\title{
Non-Fickian dispersion in open-channel flow over a porous bed
}

DOI:

10.1002/2016WR020348

\section{Document Version}

Accepted author manuscript

Link to publication record in Manchester Research Explorer

\section{Citation for published version (APA):}

Bottacin Busolin, A. (2017). Non-Fickian dispersion in open-channel flow over a porous bed. Water Resources Research, 53(8), 7426-7456. https://doi.org/10.1002/2016WR020348

\section{Published in:}

Water Resources Research

\section{Citing this paper}

Please note that where the full-text provided on Manchester Research Explorer is the Author Accepted Manuscript or Proof version this may differ from the final Published version. If citing, it is advised that you check and use the publisher's definitive version.

\section{General rights}

Copyright and moral rights for the publications made accessible in the Research Explorer are retained by the authors and/or other copyright owners and it is a condition of accessing publications that users recognise and abide by the legal requirements associated with these rights.

\section{Takedown policy}

If you believe that this document breaches copyright please refer to the University of Manchester's Takedown Procedures [http://man.ac.uk/04Y6Bo] or contact uml.scholarlycommunications@manchester.ac.uk providing relevant details, so we can investigate your claim.

\section{OPEN ACCESS}




\title{
Non-Fickian dispersion in open-channel flow over a porous bed
}

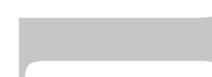

\author{
Andrea Bottacin-Busolin ${ }^{1}$
}

${ }^{1}$ School of Mechanical, Aerospace and Civil Engineering, The University of Manchester, Manchester, United Kingdom.

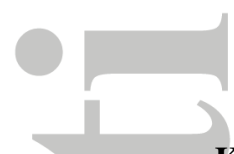

Key Points:

- Simulated concentration distributions in channel flow over a flat porous bed exhibit anomalous moment scaling and persistent skewness

- The anomalous scaling of the moments is controlled by the vertical variation of the transverse mixing coefficient in the porous bed

- The simulated concentration distributions can be approximated by beta distributions with time-varying parameters

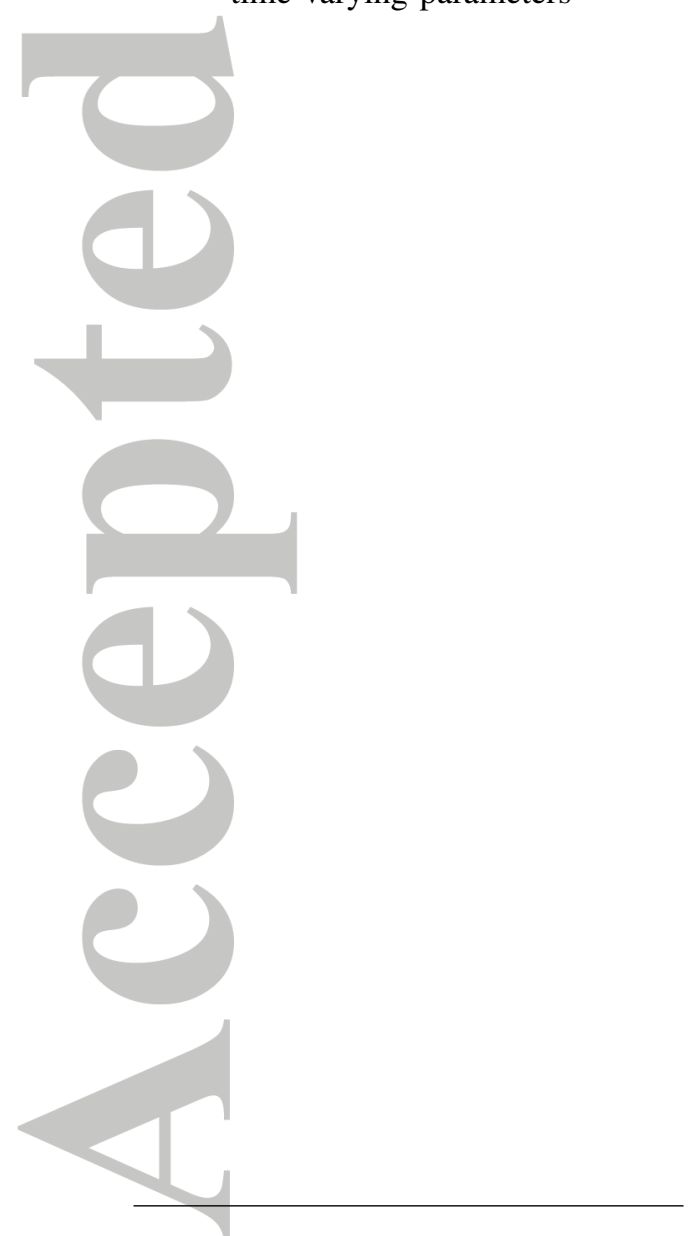

Corresponding author: A. Bottacin-Busolin, andrea.bottacinbusolin@manchester.ac.uk

This article has been accepted for publication and undergone full peer review but has not been through the copyediting, typesetting, pagination and proofreading process which may lead to differences between this version and the Version of Record. Please cite this article as an 'Accepted Article', doi: 10.1002/2016WR 020348 


\section{Abstract}

Solute transport in rivers has been traditionally represented using one-dimensional models assuming advection and Fickian dispersion along the main flow direction and transient storage in surface and subsurface dead zones. Experimental evidence from several stream tracer studies has shown that the longitudinal scaling of the moments of the breakthrough curves (BTCs) is inconsistent with classic 1-D solute transport models. In this work, simulations of advection and diffusion in a 2D and 3D channel flow over a porous bed are presented assuming an exponentially attenuated profile of the transverse mixing coefficient in the porous medium, as suggested by recent experimental and numerical studies. It is shown that the longitudinal transport in the channel is superdiffusive, and the skewness of the concentration distributions can be almost constant over a broad temporal range, with no sign of approaching zero at large times. A sensitivity analysis shows that, at large times, longitudinal dispersion is controlled by the cross-sectional profile of the in-bed transverse mixing coefficient, and by the difference between the average velocity in the channel and in the porous bed. The normalized concentration distributions can be approximated by beta-distributions with time-dependent parameters, and relationships are derived between the scaling of the parameters under power-law approximation and the scaling of the spatial and temporal moments. The results provide new insights into the physical mechanisms that control the anomalous scaling of the moments observed in field tracer studies and opens new possibilities for predictive and inverse modeling of transport processes in rivers and their catchments.

\section{Introduction}

A critical aspect of water quality modeling in streams and rivers is the prediction of solute concentrations resulting from the coupled transport of a passive tracer in the surface water and the underlying porous medium. The dispersion of solutes in natural channels has been traditionally modeled under the one-dimensional approximation, assuming that the solute is completely mixed across a transverse section of the channel. Taylor [1953, 1954] first showed that, at sufficiently large times, when fluid particles have sampled the whole cross section and become thoroughly mixed, the dispersion of a solute in a shear flow within a tube follows a Fickian diffusion law about a point moving at the mean speed of the flow. Accordingly, the spatial concentration distributions are Gaussian, and their mean and variance increase linearly with time. In a subsequent work, Aris [1956] investigated the behavior of the higher order moments of the distributions and theoretically showed that the absolute skewness of the concen- 
tration distribution tends to zero as $t^{-1 / 2}$, where $t$ is time, leading to normal distributions in the asymptotic limit of large times.

A number of later works investigated the applicability of Taylor's mixing model to openchannel flows using flume and field tracer experiments [Elder, 1959; Godfrey and Frederick, 1963; Fischer, 1966, 1967, 1968; Sayre and Chang, 1968]. Some of these experiments showed that the time-concentration distributions can be significantly more skewed than predicted by Taylor's model, raising questions on the conditions of applicability of the Fickian dispersion assumption. Deviations from the advection-dispersion equation (ADE) were noticed by Taylor himself in his first experiments on dispersion in turbulent pipe flow. Taylor [1954] initially explained these deviations as a result of the trapping of the solute into the viscous sublayer and its subsequent slow release due to molecular diffusion. This explanation was later formalized by Chatwin [1971] and Chatwin [1973] who analyzed the behavior of the variance of the concentration distributions using a two-layer formulation of the mixing process.

In the case of natural streams, the deviations are much more evident as the breakthrough curves typically exhibit a higher skewness and longer tails. This behavior was attributed by Hays [1966] to the temporary trapping of the solute in regions of small velocity and small mixing rates, such as side pockets, vegetated zones and the sediment bed. A number of 1-D formulations have been proposed to represent dead-zone processes and reproduce more accurately the skewness and the late-time behavior of tracer breakthrough curves. Among them, it is relevant to mention the dead-zone model of Hays [1966] and Pedersen [1977], based on the ADE with an additional first-order mass-transfer term, the more general Multi-Rate Mass Transfer (MRMT) formulation proposed by Haggerty et al. [2000], the Continuous Time Random Walk (CTRW) approach [Boano et al., 2007] and the generalized residence time approach of Marion et al. [2008a]. These models provide a mathematical framework in which the effect of surface and subsurface retention phenomena can be incorporated in the form of a transfer rate and a residence time distribution. Physically-based models of hyporheic exchange have been derived for different driving mechanisms such as bed-forms, bars and meanders [Boano et al., 2014] as well as side pockets [Jackson et al., 2013]. Emergent and submerged vegetation also affects solute transport by altering the distribution of the velocity and the mixing coefficient in the channel [Nepf, 2012; Shucksmith et al., 2010; Musner et al., 2014]. Battiato and Rubol [2014] and Rubol et al. [2016] used a two-domain approach to model flow through and over submerged canopies, and derived a semianalytical solution for the concentration field in submerged vegetated systems under steady-state conditions. 
Day [1975] analyzed the behavior of the first two moments of the breakthrough curves from an extensive series of tracer experiments in small mountain streams and showed that the standard deviation of an initially concentrated mass increases linearly with distance instead of its square root, in contrast with Taylor's mixing model. The data also showed that the timeconcentration distributions had a persisting skewness. In a previous experimental study, Nordin and Sabol [1974] analyzed 51 sets of tracer data covering flows from 0.85 to $6820 \mathrm{~m}^{3} \mathrm{~s}^{-1}$, and found that the variance of the temporal concentration distributions scaled as $t^{2 H}$, with $0.5 \leq$ $H \leq 1$. Some of these data was later used by Nordin and Troutman [1980] to show that, even in those cases in which the growth of the mean and the variance of the breakthrough curves was linear with time, the skewness of the distributions was roughly constant over a broad range of distances from the injection point. The behavior of the skewness was inconsistent with the prediction of a dead-zone model based on the ADE and a first-order mass transfer term.

Schmid [2002] showed that the persistent behavior of the skewness of the distributions could be explained by the passage of the tracer through a sequence of hydraulically different reaches. In particular, Schmid [2002] showed that tracer BTCs with constant skewness could be replicated by the advection-dispersion first-order mass transfer model known as Transient Storage Model (TSM) [Bencala and Walters, 1983] provided that the mean residence time in the storage zones increases with the distance from the injection point. This explanation is consistent with the experimental findings of Bottacin-Busolin et al. [2011] and Gooseff et al. [2013] indicating that the parameters of transient storage models depend on the length of the study reach. Recently, González-Pinzón et al. [2013] presented results from a meta-analysis of 384 tracer tests conducted in 44 streams around the world and brought new evidence of the anomalous scaling of the moments of the breakthrough curves. In their study, the variance of the concentration distributions was found to scale as $t^{2 H}$ with $H=0.784 \pm 0.033$, whereas the skewness was approximately constant and equal to $1.18 \pm 0.08$. In their work, González-Pinzón et al. [2013] also analyzed the scaling of the moments predicted by classic solute transport models, including the TSM, the CTRW and a Levy flight model, showing that these formulations are inconsistent with the tracer test data.

Pannone [2012] derived an exact analytical solution for the spatial moments of the concentration distributions up to the third order in a rectangular channel with impermeable boundary. Her results show that, for nonuniform initial concentration distributions, the skewness tends to slowly vanish after reaching a maximum, thus a non-negligible residual skewness can be observed at times larger than the transverse mixing time scale of the channel. The behavior 
of the moments derived by Pannone [2012] at large times is, however, consistent with a Fickian dispersion process, with variance increasing linearly with time, and skewness decreasing as $t^{-1 / 2}$. Jackson et al. [2014] investigated the effect of the geometrical complexity of a river channel using computational fluid dynamics simulations and found that the transport of a tracer along a channel with multiple lateral cavities produces a decrease in the maximum value of the coefficient of skewness (CSK) in proximity of lateral cavities, suggesting that the increase in channel complexity can decrease the gap between simulated and empirical values of CSK. Bellin et al. [2015] have recently proposed an explanation in terms of the advective hyporheic flows induced by bed-forms. Their analysis is based on the observation that there are similarities between the scaling of the moments of the BTCs reported by González-Pinzón et al. [2013] and the residence time distributions in a sediment bed predicted by an advective pumping model for a given stream-bed topography.

This work analyzes non-reactive solute transport in open-channel flow over a semi-infinite flat porous bed, and shows that a fast vanishing transverse mixing coefficient in the porous bed produces superdiffusive scaling of the moments and persistent skewness of the solute concentration distributions in the surface water. The anomalous scaling of the moments persists at times much larger than the cross-sectional mixing time scale of the channel. The results of this work provide a plausible explanation for the anomalous behavior of the solute breakthrough curves pointed out by González-Pinzón et al. [2013] and by earlier studies [Day, 1975; Nordin and Troutman, 1980], and shows that the main driver for such anomalous behavior is the attenuated profile of the transverse mixing coefficient in the porous bed. This non-Fickian behavior is found even when the bed is completely flat, and the channel uniform. Several parametric scenarios are considered, revealing a complex relationship between the behavior of the concentration distributions and the parameters that characterize the transverse diffusivity profile in the porous bed, such as the interfacial diffusivity and the attenuation depth.

The remainder of the paper is organized as follows. Section 2 presents the underlying modeling framework, which is based on the two-dimensional (2D) advection-diffusion equation, but later extended to the three-dimensional (3D) case (Section 5). Using a stochastic interpretation of the advection-diffusion process in a two-domain flow, where the two domains are given by the channel and the porous bed, it is shown that the longitudinal dispersion process in the channel can be decomposed into the longitudinal dispersion in each transport domain and a residence time distribution in the channel. This distribution depends only on the transverse diffusion process, and is independent of the velocity profile. In Section 3, the be- 
havior of the moments of the residence time in the surface water is studied using particle tracking simulations. It is shown that the moments have non-Fickian scaling and that the numerically derived distributions can be approximated by beta distributions with time dependent parameters. In Section 4, a sensitivity analysis of the effect of the velocity profile and the transverse diffusivity profile in the surface water is presented, showing that the large time behavior of the distributions is controlled by the profile of the transverse mixing coefficient in the porous bed. The results also provide insights into the effect of the velocity profile at intermediate time scales. Section 5 extends the analysis to a three-dimensional (3D) channel and further confirms the insights obtained from the two-dimensional model. Section 6 presents an asymptotic analysis of the relationship between the scaling of the spatial and temporal moments using the beta distribution with time-dependent parameters as an approximation for the normalized concentration distributions in the surface water. The beta approximation also allows for a formal proof that the hydrodynamic dispersion in the surface water is asymptotically negligible at large times compared to the spreading induced by the difference between the average velocity in the channel and the porous bed. The results and their implications for transport studies are discussed in Section 7. Finally, Section 8 summarizes the conclusions of the study and suggests ideas for future work.

\section{Two-layer Mixing Model}

The transport of a solute in a river and its sediment bed is represented here as a twolayer flow in which the upper layer represents the river channel and the lower layer represents the underlying sediment bed. The upper layer has finite depth, $h_{1}$, whereas the lower layer can have either finite or infinite depth, $h_{2}$. In the upper layer the solute is subject to relatively fast transverse mixing due to turbulence, and the flow is characterized by relatively high velocity gradients. In the lower layer, representing the porous medium, the rate of transverse mixing is significantly smaller and the velocity gradients are assumed to be negligible. The $x$-axis of the coordinate system is aligned with the direction of the bulk flow, whereas the $z$-axis is perpendicular to it and oriented upward. The origin of the $z$-axis, $z=0$, is located at the interface between the two layers so that positive values of $z$ correspond to the surface water (upper layer), and negative values to the sediment bed (lower layer). The flow velocity is everywhere parallel to the $x$-axis, and the vertical motion of a solute particle is due only to vertical diffusion. The flow properties are uniform along $x$ and depend only on the vertical coordinate, $z$. 
Consistently with the assumptions above, and restricting the analysis to the vertical $x$ -

$z$ plane, the transport of a passive tracer is described by the advection-diffusion equation:

$$
\frac{\partial c}{\partial t}+v(z) \frac{\partial c}{\partial x}=\nabla \cdot[\varepsilon(z) \nabla c]
$$

where $c(x, z)$ is the solute concentration, $v(z)$ is the flow velocity in the $x$-direction, and $\varepsilon(z)$ is the micro-dispersion (or diffusivity) tensor, which is assumed to be diagonal. Equation (1) corresponds to a stochastic process in which the position of a solute particle at time $t, \mathbf{X}(t)=$ $(X(t), Z(t))$, satisfies the following stochastic differential equations [Kitanidis, 1994; Gardiner, 2009]:

$$
\begin{aligned}
& d X_{t}=v\left(Z_{t}\right) d t+\sqrt{2 \varepsilon_{x}\left(Z_{t}\right) d t} \eta_{x, t} \\
& d Z_{t}=\left.\frac{d \varepsilon_{z}}{d z}\right|_{z=Z_{t}} d t+\sqrt{2 \varepsilon_{z}\left(Z_{t}\right) d t} \eta_{z, t}
\end{aligned}
$$

where $\eta_{x, t}$ and $\eta_{z, t}$ are independent normally distributed random variables, and $\varepsilon_{x}$ and $\varepsilon_{z}$ are the longitudinal and vertical mixing coefficients, respectively.

The longitudinal spreading of the solute in the river channel is described by the distribution $\psi\left(x, t \mid \mathbf{x}_{0}\right)$ representing the conditional density of $X$ at time $t$ given that $\mathbf{X}=\mathbf{x}_{0}$ at $t=$ 0 and that $Z>0$ at time $t$. The latter condition implies that the particle is in the surface water at time $t$, which is where solute concentrations are typically measured in tracer tests. It is assumed that the probability density function of the longitudinal distance travelled by a particle during its motion along one of the layers depends only on the time spent in the same layer, $T_{i}$, where $i=1$ for the upper layer and $i=2$ for the lower layer. This assumption implies that either the vertical velocity profile is uniform in the upper layer, or transverse mixing across the flow depth is achieved rapidly compared to the lower layer, so that the correlation between longitudinal particle displacement in the upper layer and vertical particle position can be neglected for a given residence time in the same layer. The assumption can also be considered acceptable for deep sediment beds as long as the longitudinal spreading induced by vertical velocity gradients in the bed is small compared to the spreading induced by the different average velocity in the surface water (upper layer) and in the sediment bed (lower layer). The distribution of the distance travelled in each layer knowing that a particle has spent a time $t_{i}$ in the same layer is denoted by $g_{i}\left(x \mid t_{i}\right)$, where $i=1$ for the upper layer and $i=2$ for the lower layer.

The conditional density of the residence time in the upper layer, $T_{1}$, at time $t$ given that the particle is in the upper layer $(Z>0)$ at time $t$ is denoted by $f\left(t_{1}, t \mid z_{0}\right)$. Consistently with 
the assumptions on the velocity distribution and the transverse mixing coefficient, the residence time in the upper layer does not depend on the longitudinal position of the injection point, $x_{0}$.

Using the residence time function $f$, the conditional density $\psi\left(x, t \mid \mathbf{x}_{0}\right)$ can be derived from the two densities $g_{1}\left(x \mid t_{1}\right)$ and $g_{2}\left(x \mid t_{2}=t-t_{1}\right)$ :

$$
\psi\left(x, t \mid \mathbf{x}_{0}\right)=\int_{-\infty}^{\infty} \int_{0}^{\infty} g_{1}\left(x-x_{0}-x^{\prime} \mid t_{1}\right) g_{2}\left(x^{\prime} \mid t-t_{1}\right) f\left(t_{1}, t \mid z_{0}\right) d t_{1} d x^{\prime}
$$

As a special case, if the longitudinal transport in each layer is Fickian, the distributions $g_{1}$ and $g_{2}$ can be derived from the solution of the classic advection-dispersion equation for an instantaneous point injection:

$$
g_{i}(x \mid t)=\frac{1}{2 \sqrt{\pi D_{i} t}} \exp \left[-\frac{\left(x-u_{i} t\right)^{2}}{4 D_{i} t}\right]
$$

where $u_{i}$ and $D_{i}$ are the average velocity and longitudinal dispersion coefficient in the $i$-the layer $(i=1,2)$, respectively. This representation is consistent with the assumption that the transport in the two layers is characterized by two distinct transverse mixing rates and velocity profiles, and that mixing in the upper layer is much faster than mixing in the lower layer.

In case of a constant velocity in the upper and the lower layer, the conditional density of the distance travelled in each layer is given by $g_{i}\left(x \mid t_{i}\right)=\delta\left(x-u_{i} t_{i}\right)$, where $\delta(x)$ is the Dirac delta function. Accordingly, the relationship between the total longitudinal distance travelled by a solute particle and the time spent in the upper layer is given by

$$
X=x_{0}+u_{1} T_{1}+u_{2}\left(t-T_{1}\right)=x_{0}+u_{2} t+\left(u_{1}-u_{2}\right) T_{1}
$$

and therefore, from the transformation law of random variables,

$$
\psi\left(x, t \mid \mathbf{x}_{0}\right)=\frac{1}{u_{1}-u_{2}} f\left(\frac{x-x_{0}-u_{2} t}{u_{1}-u_{2}}, t \mid z_{0}\right)
$$

Equation (7) shows that the distribution of $X$ for the particles in the surface water can be inferred from the distribution $f\left(t_{1}, t \mid z_{0}\right)$.

For an instantaneous release of a mass of solute $M$ at point $\mathbf{x}_{0}$ at time $t=0$, the relation between the distribution $\psi\left(x, t \mid \mathbf{x}_{0}\right)$ and the cross-sectionally averaged concentration in the surface water is

$$
c\left(x, t \mid \mathbf{x}_{0}\right)=\frac{M}{A} p\left(t \mid z_{0}\right) \psi\left(x, t \mid \mathbf{x}_{0}\right)
$$

where $A$ is the cross sectional area of the channel and $p\left(t \mid z_{0}\right)$ represents the probability that a solute particle is in the surface water $(Z>0)$ at time $t$. As the function $f$, the probability $p\left(t \mid z_{0}\right)$ depends only on the transverse mixing process. Since $\int_{-\infty}^{\infty} \psi\left(x, t \mid \mathbf{x}_{0}\right) d x=1$, the in- 
tegral of the spatial concentration distribution for an instantaneous injection is

$$
\int_{\infty}^{\infty} c\left(x, t \mid \mathbf{x}_{0}\right) d x=\frac{M}{A} p\left(t \mid z_{0}\right)
$$

which implies that the PDF $\psi\left(x, t \mid \mathbf{x}_{0}\right)$ is equivalent to the spatial concentration distribution normalized by the area under the curve.

\section{Residence Time in the Surface Water}

\subsection{Numerical Simulations}

The conditional density of the residence time in the upper layer $f\left(t_{1}, t \mid z_{0}\right)$ can be derived from random walk simulations of the transverse mixing process across a vertical section of the flow domain, comprising the surface water (upper layer) and the porous medium (lower layer). This requires the assumption of a vertical profile of the vertical diffusion coefficient, $\varepsilon(z) \equiv \varepsilon_{z}(z)$. The coefficient $\varepsilon$ accounts for turbulent diffusion in the surface water, mechanical dispersion in the porous medium, and molecular diffusion.

To generalize the numerical results, it is convenient to work with the nondimensional quantities:

$$
\hat{z}=\frac{z}{h_{1}}, \quad \hat{t}=\frac{t}{\tau_{1}}, \quad \hat{\varepsilon}=\frac{\varepsilon}{\varepsilon_{1}}
$$

where $h_{1}$ is the surface water depth (i.e. thickness of the upper layer), $\tau_{1}$ is the Taylor vertical mixing scale in the surface water, defined as

$$
\tau_{1}=\frac{h_{1}^{2}}{\varepsilon_{1}}
$$

and $\varepsilon_{1}$ is the average transverse diffusivity in the surface water,

$$
\varepsilon_{1}=\frac{1}{h_{1}} \int_{0}^{h_{1}} \varepsilon(z) d z
$$

The eddy diffusivity in a turbulent boundary layer is strictly related to the eddy viscosity, $\nu_{t}$, and is generally taken as $\varepsilon=\nu_{t} / S_{n}$, where $S_{n}$ is the dimensionless turbulent Schmidt number [Rodi, 1993]. For a turbulent flow over an impermeable bed, the eddy diffusivity can be shown to follow a parabolic profile [Fischer et al., 1979]. For a turbulent flow over a porous bed, experimental results by Ferreira et al. [2010] and numerical results by Breugem et al. [2006] suggest that the diffusivity still resembles a parabolic curve in the surface water, but with a smooth transition to an exponentially attenuated diffusivity profile in the porous bed [Chandler et al., 2016]. A possible parametrization of the transverse diffusivity profile that complies 
with this qualitative behavior is the following:

$$
\hat{\varepsilon}(\hat{z})= \begin{cases}\sum_{n=0}^{5} b_{i} \hat{z}^{n}, & 0<\hat{z} \leq 1 \\ \hat{\varepsilon}_{\infty}+\left(\hat{\varepsilon}_{0}-\hat{\varepsilon}_{\infty}\right) e^{\hat{z} / \hat{h}_{\infty},} & \hat{z} \leq 0\end{cases}
$$

where $\hat{\varepsilon}_{0}=\varepsilon_{0} / \varepsilon_{1}$ is the interface diffusion coefficient, $\hat{h}_{\infty}=h_{\infty} / h_{1}$ is an attenuation depth

at which $\hat{\varepsilon}$ approaches $\hat{\varepsilon}_{\infty}=\varepsilon_{\infty} / \varepsilon_{1}$, and where the polynomial coefficients $b_{i}$ for the surface water profile satisfy the conditions:

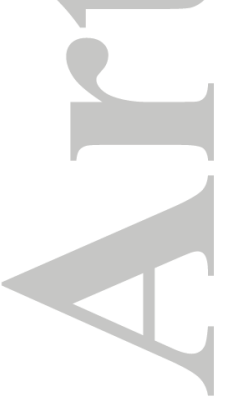

$$
\begin{aligned}
\hat{\varepsilon}=\hat{\varepsilon}_{0} & \text { at } \hat{z}=0 \\
\hat{\varepsilon}=0 & \text { at } \hat{z}=1 \\
\frac{d \hat{\varepsilon}}{d \hat{z}}=\frac{\hat{\varepsilon}_{0}-\hat{\varepsilon}_{\infty}}{\hat{h}_{\infty}} & \text { at } \hat{z}=0 \\
\frac{d \hat{\varepsilon}}{d \hat{z}}=-6 & \text { at } \hat{z}=1 \\
\frac{d \hat{\varepsilon}}{d \hat{z}}=0 & \text { at } \hat{z}=1 / 2
\end{aligned}
$$

together with the normalization condition (12). The analytical expressions of the coefficients $b_{i}$ are given in Appendix A: (equations (A.1)-(A.6)). The conditions above ensure that the diffusivity profile closely follows a parabolic profile near the surface, and that the diffusivity is continuous and differentiable at the interface between the upper and the lower layer. The coefficients $b_{i}$ can, however, be adapted to represent a broader range of profiles such as those presented by Ferreira et al. [2010]. Alternative parametrizations are possible, but it will be shown in the sensitivity analysis presented in Section 4 that the exact form of the vertical diffusivity profile in the surface water has negligible impact on the concentration distributions at times larger than the vertical mixing scale. This is in line with a long tradition of studies that have assumed the transverse mixing coefficient to be uniform in the channel. The vertical profile of the diffusion coefficient described by Equation (13) is shown in Figure 1 for some of the parametric scenarios in Table 1 together with some other diffusivity profiles that will be considered later in the sensitivity analysis presented in Section 4.

Figure 1. Vertical profile of transverse diffusivity obtained from equation (13) for different values of the parameters $\hat{\varepsilon}_{0}, \hat{\varepsilon}_{\infty}$ and $\hat{h}_{\infty}$ reported in Table 1 . The parabolic profile (dashed red line) represents the case of open channel flow over an impermeable bed.

The assumption of an exponentially attenuated profile of the transverse mixing coefficient in the transitional zone between the sediment-water interface and the underground flow 
Table 1. Parameters used in the random walk simulations of transverse mixing.

\begin{tabular}{llllll}
\hline Run No. & $\hat{\varepsilon}(z)$ & $\hat{\varepsilon}_{0}$ & $\hat{\varepsilon}_{\infty}$ & $\hat{h}_{\infty}$ & $\hat{h}_{2}$ \\
\hline Run A-1 & Constant, $\hat{\varepsilon}=1$ & - & - & - & 1 \\
Run A-2 & Equation (13) & 0.1 & 0 & 0.5 & $\infty$ \\
Run A-3 & Equation (13) & 0.03 & 0 & 0.3 & $\infty$ \\
Run A-4 & Equation (13) & $10^{-3}$ & 0 & 0.02 & $\infty$ \\
Run A-5 & Equation (13) & $10^{-3}$ & 0 & 0.01 & $\infty$ \\
Run A-6 & Equation (13) & $10^{-3}$ & $10^{-3}$ & 0.02 & $\infty$ \\
Run A-7 & Equation (13) & $10^{-3}$ & $10^{-4}$ & 0.02 & $\infty$ \\
Run A-8 & Equation (13) & $10^{-4}$ & 0 & 0.02 & $\infty$ \\
Run A-9 & Equation (13) & $10^{-4}$ & 0 & 0.01 & $\infty$ \\
\hline
\end{tabular}

induced by the stream gradient is consistent with the empirical equations of Chandler et al.

[2016] linking flow shear velocity, bed permeability and mean sediment particle diameter with the transverse mixing coefficient in a porous bed below a turbulent flow. It is also consistent with the exponential velocity attenuation found experimentally by Leu et al. [2009], and via direct numerical simulation (DNS) by Breugem et al. [2006]. Equation (13) provides a plau-

sible representation of the vertical variation of the mixing coefficient in the surface water and the porous medium, and allows sufficient flexibility to get a qualitative understanding of how the traverse diffusivity profile affects the distribution $f\left(t_{1}, t \mid z_{0}\right)$.

The residence time function $f\left(t_{1}, t \mid z_{0}\right)$ is obtained from random walk simulations of the vertical mixing induced by the diffusivity profile (13). In the simulations, the vertical position of a particle $Z_{t}$ is described by the following stochastic differential equation:

$$
d \hat{Z}_{t}=\left.\frac{d \hat{\varepsilon}}{d \hat{z}}\right|_{\hat{z}=\hat{Z}_{t}} d \hat{t}+\sqrt{2 \hat{\varepsilon}\left(\hat{Z}_{t}\right) d \hat{t}} \eta_{t}
$$

where $\eta_{t}$ is an independent standard Gaussian random variable. The first term in equation (19) represents the additional drift associated with the spatial variability of the vertical mixing coefficient [Kitanidis, 1994], which is assumed to be a continuous and differentiable function of $\hat{z}$. Note that the residence time in the surface water, $T_{1}$, does not depend on the longitudinal particle displacements, and therefore the function $f$ does not dependent on equation (2) and the velocity profile $v(z)$. Equation (19) is solved using the weak order 2.0 predictor-corrector method described by Platen [1995] [Platen and Bruti-Liberati, 2010, see also]. 
The motion of $10^{4}$ particles released at the top boundary of the upper layer, $\hat{z}=1$, at $\hat{t}=0$ was simulated using a time step $\Delta \hat{t}=10^{-3}$. This time step ensures convergence of the numerical results, as demonstrated by a series of numerical tests. Simulations were conducted for several combinations of the parameters of the vertical diffusivity profile, as reported in Table 1. In particular, Run A-1 represents the case of a constant diffusivity profile across the two layers, corresponding to an overall vertical mixing time scale for the combined layers of $\left(2 h_{1}\right)^{2} / \varepsilon_{1}=$ $4 \tau_{1}$. This case is not representative of any real conditions, but is presented as a reference for comparison with the other runs. Runs A-2 and A-3 are characterized by relatively high interfacial diffusivities, $\hat{\varepsilon}_{0}$, and high attenuation depths, $\hat{h}_{\infty}$, as opposed to Runs A-4 to A-9 which have relatively small $\hat{\varepsilon}_{0}$ and $\hat{h}_{\infty}$. The values of the attenuation depth in Runs A-4 to A-9 range between 0.01 and 0.02, in line with the experimental results of Chandler et al. [2016] whose empirical equations for the in-bed transverse mixing coefficient imply $\hat{h}_{\infty}=1 / 55 \approx 0.018$. In natural watercourses the value of the attenuation depth is expected to be higher due to interactions between flow and bed topography and heterogeneities which enhance transverse mixing [Hester et al., 2017].

From the numerical simulations it is possible to obtain an estimate of the normalized density $\hat{f}\left(\hat{t}_{1}, \hat{t} \mid \hat{z}_{0}\right)$, defined implicitly as

$$
f\left(t_{1}, t \mid z_{0}\right)=\frac{1}{\tau_{1}} \hat{f}\left(\frac{t_{1}}{\tau_{1}}, \frac{t}{\tau_{1}} \mid \frac{z_{0}}{h}\right)
$$

It can be convenient, though, to visualize the results in terms of the $\operatorname{PDF} f^{*}\left(t_{1}^{*}, \hat{t} \mid \hat{z}\right)$ of the normalized time in the surface water $T_{1}^{*}=T_{1} / t$, such that

$$
\hat{f}\left(\hat{t}, \hat{t} \mid \hat{z}_{0}\right)=\frac{1}{\hat{t}} f^{*}\left(\frac{\hat{t}_{1}}{\hat{t}}, \hat{t} \mid \hat{z}_{0}\right)
$$

Since $0 \leq T_{1}^{*} \leq 1$, the function $f^{*}\left(t_{1}^{*}, \hat{t} \mid \hat{z}\right)$ can take non-zero values only in the interval $0 \leq$ $t_{1}^{*} \leq 1$, and hence $f^{*} \equiv 0$ for $t_{1}^{*}<0$ or $t_{1}^{*}>1$.

The raw moments of $f$ are given by:

$$
m_{T_{1}, n}^{\prime}\left(t \mid z_{0}\right)=\int_{-\infty}^{\infty} t_{1}^{n} f\left(t_{1}, t \mid z_{0}\right) d t_{1}
$$

from which the second and third central moments can be derived as follows [Kenney and Keeping, 1947; Pannone, 2012]:

$$
\begin{aligned}
& m_{T_{1}, 2}=\int_{-\infty}^{\infty}\left(t_{1}-m_{T_{1}, 1}\right)^{2} f\left(t_{1}, t \mid z_{0}\right) d t_{1}=m_{T_{1}, 2}^{\prime}-\left(m_{T_{1}, 1}\right)^{2} \\
& m_{T_{1}, 3}=\int_{-\infty}^{\infty}\left(t_{1}-m_{T_{1}, 1}\right)^{3} f\left(t_{1}, t \mid z_{0}\right) d t_{1}=m_{T_{1}, 3}^{\prime}-3 m_{T_{1}, 1} m_{T_{1}, 2}^{\prime}+2 m_{T_{1}, 1}^{3}
\end{aligned}
$$


where $m_{T_{1}, 1}=m_{T_{1}, 1}^{\prime}$. The coefficient of skewness (CSK) of $T_{1}$ is defined as:

$$
\gamma_{T_{1}}=\frac{m_{T_{1}, 3}}{\left(m_{T_{1}, 2}\right)^{3 / 2}}
$$

\subsection{Results}

The discrete PDFs derived from Runs A-1 and A-3 (Table 1) with $\hat{z}_{0}=1$ are plotted as light-colored dashed lines in Figure 2 for different simulation times, $\hat{t}$. The shape of the numerical distributions and the fact that the function $f^{*}$ has compact support in the interval $0 \leq$ $t_{1}^{*} \leq 1$ suggest that a suitable approximation for $f^{*}$ is the beta PDF:

$$
f^{*}\left(t_{1}^{*}, \hat{t} \mid \hat{z}_{0}=1\right)=\frac{1}{a \mathrm{~B}(\alpha, \beta)}\left(\frac{t_{1}^{*}}{a}\right)^{\alpha-1}\left(1-\frac{t_{1}^{*}}{a}\right)^{\beta-1}
$$

where $a, \alpha$ and $\beta$ are parameters of the distribution, and $\mathrm{B}(\alpha, \beta)$ is the beta function defined as:

$$
\mathrm{B}(\alpha, \beta)=\int_{0}^{1} \nu^{\alpha-1}(1-\nu)^{\beta-1} d \nu
$$

where $\nu$ is a dummy variable. Compared to the standard beta distribution, the additional scale parameter $a$ in equation (26) allows to scale the interval on which the function is non-zero. The approximating beta distributions obtained by least-square fit of the simulation results are plotted as continuous lines in Figure 2. In general, the parameters $\alpha, \beta$ and $a$ depend on the vertical profile of the mixing coefficient, $\varepsilon(z)$, the thickness of the sediment layer, the elevation of the point at which solute is released, $z_{0}$, and time, $t$.

Figure 2. Dimensionless distribution $f^{*}\left(t_{1}^{*}, \hat{t} \mid \hat{z}_{0}=1\right)$ of the residence time $t_{1}^{*}=t_{1} / t$ in the surface water given that a particle is in the surface water at time $\hat{t}=t / \tau_{1}$. The dashed lines represent the discrete PDF obtained from the numerical simulations, whereas the continuous lines represent the approximating beta distribution (26). Panel (a) represents the case in which the porous bed (lower layer) has finite depth equal to that of the upper layer, $\hat{h}_{2}=1$, and the transverse diffusion coefficient is constant, $\hat{\varepsilon}=1$. Panel (b) represents the case in which the bed (lower layer) is semi-infinite, and the transverse diffusion coefficient is a function of $\hat{z}$ according to equation (13).

Figure 2 shows that the conditional density of the normalized residence time in the surface water, $f^{*}$, is significantly different in Run A-1 and A-3. Run A-1 (Figure 2a) represents the case of two layers with uniform diffusivity and the same thickness. This case is characterized by a first initial phase in which the distribution is negatively skewed towards $t_{1}^{*}=1$, 
and becomes gradually a symmetric bell-shaped distribution centered around $t_{1}^{*}=0.5$, with decreasing standard deviation as the time increases. This behavior follows from the fact that, since the solute is released at $\hat{z}_{0}=1$, the solute is initially concentrated in the upper layer and gradually mixes across the two layers of finite depths until complete mixing is achieved. Under this condition, the average fraction of time spent in layer $i$, where $i=1$ for the surface water (upper layer) and $i=2$ for the sediment bed (lower layer), is equal to $h_{i} /\left(h_{1}+h_{2}\right)$. The decrease in the standard deviation of $T_{1}^{*}=T_{1} / t$ after a relatively fast increase in the initial mixing phase implies that the standard deviation of the residence time $T_{1}$ increases more slowly than the time elapsed from injection, $t$.

Run A-3 (Figure 2b) corresponds to the case of a semi-infinite porous bed in which the transverse diffusivity decreases exponentially with depth. It can be seen that in this case the variance decreases with a much smaller rate compared to Run A-1, and a certain skewness can be observed even at long times after the injection. It can also be observed that there is a cutoff value of the normalized time in the surface water $t_{1}^{*}$ for which the corresponding probability is virtually zero. The behavior of the moments of this function and their relationship with the spatial moments of the transition PDF $\psi\left(x, t \mid \mathbf{x}_{0}\right)$ will be investigated in Section 6 .

The moments of the function $\hat{f}\left(\hat{t}_{1}, \hat{t} \mid \hat{z}_{0}=1\right)$ obtained by numerical particle tracking are plotted in Figure 3 for the different scenarios presented in Table 1. Figure 3a shows the fraction of mass in the upper layer as a function of time, $p\left(\hat{t} \mid \hat{z}_{0}=1\right)$. For Run A-1, the mass in the upper layer decreases very rapidly, and within a time period slightly longer than the vertical mixing scale it reaches the asymptotic value of 0.5 , indicating an equal partition of the mass in the two layers. For the other runs, mass decreases continuously without reaching a final asymptotic value as the solute continues to be transferred from the upper layer to the lower layer of infinite depth. Figure 3 also shows that the rate at which the mass in the upper layer is transferred to the lower layer is higher for higher $\hat{\varepsilon}_{\infty}, \hat{\varepsilon_{0}}$ and $\hat{h}_{\infty}$.

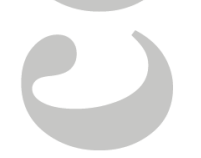

Figure 3. (a) Fraction of the total injected mass in the surface water (upper layer), $p$, versus time, $\hat{t}$; (b) mean $m_{T_{1}, 1}$, (c) variance $m_{T_{1}, 2}$, and (d) coefficient of skewness (CSK) $\gamma_{T_{1}}$ of the residence time in the surface water (upper layer) $T_{1}$ for the particles in the surface water at time $\hat{t}$. For uniform flow in the two layers, the moments of $T_{1}$ are related to the spatial moments of the concentration distributions by equations (28) and $\gamma_{X}=\gamma_{T_{1}}$ 
Figure $3 \mathrm{~b}$ shows the behavior of the first central moment of the residence time function $f$. It is observed that for Run A-1, the moment $m_{T_{1}, 1}$ tends to increase linearly with time, indicating that $m_{X, 1}$ is also a linear function of time. However, a slight sublinear behavior can be observed in Runs A-2 and A-3. The behavior of the second central moment of $T_{1}$ is shown in Figure 3c. It can be seen that $m_{T_{1}, 2}$ increases linearly with time in Run A-1 after a time equal to the vertical mixing time scale for the combined layers $(\hat{t}=4)$, whereas the other runs follow a superlinear power-law trend scaling as $t^{2}$. Intermediate scaling exponents between 1.5 and 2 are observed in Runs A-2 and A-3.

The behavior of the skewness is presented in Figure 3d, which shows significant differences between the simulations. At small times from injection, the distributions have a high negative skewness, because most of the particles in the surface water (upper layer) at time $\hat{t}$ have spent most of the time in the surface water. In Run A-1 $\left(\hat{h}_{2}=1\right.$ and $\left.\hat{\varepsilon}=1\right)$ the absolute value of the coefficient of skewness decreases as $t^{-1 / 2}$ after an initial phase characterized by a faster decrease. In Runs A-2 to A-4 ( $\hat{h}_{2}=\infty$, and $\left.\hat{\varepsilon}_{\infty}=0, \hat{\varepsilon}_{\infty}=0\right)\left|\gamma_{T_{1}}\right|$ tend to approach a constant value after an initial decreasing phase. Run A-6, characterized by a constant diffusivity in the bed $\left(\hat{\varepsilon}_{0}=\hat{\varepsilon}_{\infty}=10^{-3}\right)$, decreases monotonically according to a power-law that is approximately proportional to $t^{-1 / 2}$, whereas Run A-7, with interface diffusivity $\hat{\varepsilon}_{0}=10^{-3}$ and asymptotic diffusivity in the bed $\hat{\varepsilon}_{\infty}=10^{-4}$, closely follows the curve obtained for Run A4 in the initial phase, but then diverges from it at longer times. Comparison between Run A2, A-3, A-4 and A-5 shows that smaller values of $\hat{h}_{\infty}$ are associated with higher skewness, whilst the mass transfer to the bed is slower (Figure 3a). This implies that a deeper transfer into the bed is not necessarily associated with higher skewness of the concentration distributions. In Runs A-8 and A-9 the interfacial diffusivity is an order of magnitude smaller than in Runs A-4 and A-5, whereas $\hat{h}_{\infty}$ in Run A-8 is the same as in Run A-4, and $\hat{h}_{\infty}$ in Run A9 is the same as in A-5. Comparison between these runs shows that smaller interfacial diffusivities are associated with higher skewness for $\hat{t}>1$, but the large time value of the skewness appears to be controlled by $\hat{h}_{\infty}$.

\section{Sensitivity Analysis}

The validity of the decomposition (4) and the approximation (7) linking the function $f\left(t_{1}, t \mid z_{0}\right)$ to $\psi\left(x, t \mid z_{0}\right)$ can be examined by assuming a velocity profile in the surface water. The diffusivity profile is strictly related to the eddy viscosity profile, which in turn depends on the velocity distribution. In general, the diffusivity and velocity distributions would have to be de- 
termined by computational fluid dynamics (CFD) simulation [e.g. Breugem et al., 2006]. However, since the aim of the sensitivity analysis presented in this section is to examine how the velocity profile may affect the behavior of the concentration distributions at large times, it will be sufficient to assume a velocity profile that complies with some of the expected properties of turbulent open-channel flows over a porous bed. Parameters can then be varied to examine the range of applicability of equation (7) derived for uniform velocities in the channel and the porous bed. Consistently with equation (7), the relationship between the central moments of $X^{\prime}=X-x_{0}-u_{2} t$ and the moments of $T_{1}$ is:

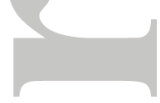

$$
m_{X^{\prime}, n}=u^{n} m_{T_{1}, n}
$$

where $n=1,2, \ldots$ and $u=u_{1}-u_{2}$. Note $m_{X^{\prime}, n}=m_{X, n}$ for $n=2,3, \ldots$ The range of applicability of (7) can therefore be analyzed by comparing the spatial moments derived from particle tracking simulations of longitudinal dispersion for a prescribed velocity profile, and the moments derived from $T_{1}$ according to (28).

The velocity profile in a rough turbulent boundary layer can be approximated by a powerlaw [Chanson, 2004], whereas the velocity profile is known to be exponentially attenuated in the sediment bed [Breugem et al., 2006; Leu et al., 2009]. This suggests the following parameterization for the velocity profile:

$$
\hat{v}(\hat{z})= \begin{cases}\frac{1+n_{u}}{n_{u}}\left(1-\hat{v}_{0}\right) z^{1 / n_{u}}+\hat{v}_{0} & \text { for } 0<\hat{z} \leq 1 \\ \hat{v}_{\infty}+\left(\hat{v}_{0}-\hat{v}_{\infty}\right) e^{\hat{z} / \hat{h}_{u}}, & \text { for } \hat{z} \leq 0\end{cases}
$$

where $\hat{v}_{0}=v_{0} / u_{1}$ is the interface velocity, $\hat{v}_{\infty}=v_{\infty} / u_{1}$ is the velocity of the underground flow, $\hat{h}_{u}=h_{u} / h_{1}$ is the velocity attenuation depth in the sediment, and $u_{1}$ is the average velocity in the channel, which in the $2 \mathrm{D}$ case is given by

$$
u_{1}=\frac{1}{h_{1}} \int_{0}^{h_{1}} v(z) d z
$$

The exponent $n_{u}$ is related to the flow resistance, and can be expressed for uniform equilibrium flows as $n_{u}=K \sqrt{8 / \phi}$, where $K$ is the von Karman constant and $\phi$ is the Darcy friction factor [Chen, 1991; Chanson, 2004]. The distribution (29) ensures a continuous transition at the sediment-water interface with interfacial velocity $v_{0}$, and satisfies the normalization condition (30).

Since the longitudinal spreading induced by the microscale diffusivity $\varepsilon_{x}$ in the $x$-direction is generally orders of magnitude smaller than the spreading induced by the velocity gradients [Fischer et al., 1979], its contribution to the longitudinal transport process at large times can 
Table 2. Parameters used in the analysis of the effect of the velocity profile described by equation (29) and using the vertical diffusivity profile (13).

\begin{tabular}{clllllll}
\hline Run No. & $\hat{\varepsilon}_{0}$ & $\hat{\varepsilon}_{\infty}$ & $\hat{h}_{\infty}$ & $n_{u}$ & $\hat{v}_{0}$ & $\hat{v}_{\infty}$ & $h_{u}$ \\
\hline Run C-1 & $10^{-3}$ & 0 & 0.02 & 7 & 0 & 0 & - \\
Run C-2 & $10^{-3}$ & 0 & 0.02 & 5 & 0 & 0 & - \\
Run C-3 & $10^{-3}$ & 0 & 0.02 & 2 & 0 & 0 & - \\
Run C-4 & $10^{-3}$ & 0 & 0.02 & 7 & $10^{-1}$ & 0 & 0.02 \\
Run C-5 & $10^{-3}$ & 0 & 0.02 & 7 & $10^{-2}$ & 0 & 0.02 \\
\hline
\end{tabular}

be neglected. Accordingly, the stochastic differential equations representing the position of a solute particle at time $\hat{t}$ are, in nondimensional form:

$$
\begin{aligned}
& d \hat{X}_{t}=\hat{v}\left(\hat{Z}_{t}\right) d \hat{t} \\
& d \hat{Z}_{t}=\left.\frac{d \hat{\varepsilon}}{d \hat{z}}\right|_{\hat{z}=\hat{Z}_{t}} d \hat{t}+\sqrt{2 \hat{\varepsilon}\left(\hat{Z}_{t}\right) d \hat{t}} \eta_{t}
\end{aligned}
$$

where $\hat{X}_{t}=X_{t} / \ell_{1}$ and $\ell_{1}=u_{1} \tau_{1}$. Note that longitudinal distances are normalized by $\ell_{1}$ whereas tranšverse (vertical) distances are normalized by $h_{1}$.

The results presented in Figure 4 for the parametric scenarios in Table 2 show that the spatial moments of the concentration distribution converge to those predicted from the moments of the residence time in the surface water according to (28) within a few multiples of the vertical mixing time scale, $\tau_{1}$. This implies that longitudinal dispersion in the channel is controlled by the profile of the transverse mixing coefficient and by the difference between the average velocity in the channel, $u_{1}$, and in the bed, $u_{2}$. Since generally $u_{2} \ll u_{1}$, it can be further assumed that $u_{2} \approx 0$.

Figure 4. Spatial moments $m_{X, n}, n=1,2$, and CSK for the parametric scenarios in Table 2. The dashed lines represent the curves obtained from particle tracking simulations of $X$ whereas the continuous lines are obtained from the moments of $T_{1}$ according to (28) using particle tracking simulations of transverse mixing only. Different runs correspond to different velocity profiles.

A sensitivity analysis was also carried out to examine how the assumptions on $\varepsilon(z)$ affect the moments of the concentration distributions. To this end, instead of approximating $\varepsilon(z)$ with (13), the diffusivity is taken as constant in the surface water except in the near-bed re- 
gion where the diffusivity experiences a smooth transition to the exponentially attenuated pro-

file in the sediment bed. Denoting by $\hat{z}_{m}=z_{m} / h$ is the nondimensional blend height, an expression that ensures continuity of the diffusivity profile up to the first order derivative is the following:

$$
\hat{\varepsilon}(\hat{z})= \begin{cases}\hat{\varepsilon}_{s} & \hat{z}_{m} \leq \hat{z} \leq 1 \\ \sum_{n=0}^{3} b_{i} \hat{z}^{n} & 0<\hat{z}<\hat{z}_{m} \\ \hat{\varepsilon}_{\infty}+\left(\hat{\varepsilon}_{0}-\hat{\varepsilon}_{\infty}\right) e^{\hat{z} / \hat{h}_{\infty}} & \hat{z} \leq 0\end{cases}
$$

where the polynomial coefficients $b_{i}$ and the parameter $\hat{\varepsilon}_{s}$ are determined by imposing:

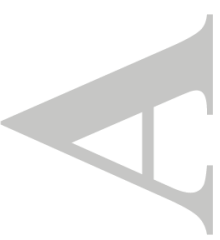

$$
\begin{aligned}
\hat{\varepsilon}=\hat{\varepsilon}_{0} & \text { at } \hat{z}=0 \\
\frac{d \hat{\varepsilon}}{d \hat{z}}=\frac{\hat{\varepsilon}_{0}-\hat{\varepsilon}_{\infty}}{\hat{h}_{\infty}} & \text { at } \hat{z}=0 \\
\hat{\varepsilon}=\hat{\varepsilon}_{s} & \text { at } \hat{z}=\hat{z}_{m} \\
\frac{d \hat{\varepsilon}}{d \hat{z}}=0 & \text { at } \hat{z}=\hat{z}_{m}
\end{aligned}
$$

and the normalization condition (12). The analytical expressions of the coefficients $b_{i}$ and the parameter $\hat{\varepsilon}_{s}$ are given in Appendix A: (equations (A.12)-(A.16)). Examples of the profiles generated by equation (33) for some of the parametric scenarios presented in Table 3 are shown in Figure 1.

The simulated moments and skewness of the concentration distributions are presented in Figure 5 for the scenarios in Table 3. The results show that, for $t>\tau_{1}$, there is no significant difference between the moments obtained from (33) and the moments obtained from (13), even when the blend height, $z_{m}$, is varied from 0.1 to 0.5 . This means that, at times larger than the vertical mixing time scale, the behavior of the moments is controlled by the average transverse diffusivity in the surface water, $\varepsilon_{1}$, and the profile $\varepsilon(z)$ in the sediment bed. An accurate description of the diffusivity profile in the surface water does not appear to be necessary to describe the large time behavior of the concentration distributions.

Figure 5. Spatial moments $m_{X, n}, n=1,2$, and CSK for the parametric scenarios in Table 3 . The curves are obtained from the moments of $T_{1}$ according to (28) using particle tracking simulations of transverse mixing only. Different runs correspond to different profiles of the transverse diffusivity profile in the surface water. 
Table 3. Parameters used in the analysis of the effect of the vertical diffusivity profile in the surface water.

\begin{tabular}{llllll}
\hline Run No. & $\hat{\varepsilon}(\hat{z})$ & $\hat{\varepsilon}_{0}$ & $\hat{\varepsilon}_{\infty}$ & $\hat{h}_{\infty}$ & $\hat{z}_{m}$ \\
\hline Run D-1 & Equation (13) & $10^{-3}$ & 0 & 0.02 & - \\
Run D-2 & Equation (33) & $10^{-3}$ & 0 & 0.02 & 0.1 \\
Run D-3 & Equation (33) & $10^{-3}$ & 0 & 0.02 & 0.3 \\
Run D-4 & Equation (33) & $10^{-3}$ & 0 & 0.02 & 0.5 \\
\hline
\end{tabular}

\section{Dispersion in a 3D Channel}

The two-dimensional (2D) analysis presented in Section 2 can be extended to a threedimensional (3D) channel. The two-domain decomposition expressed by equation (4) is still valid, with the function $f\left(t_{1}, t \mid z_{0}, y_{0}\right)$ now representing the conditional density of the residence time in the channel, $T_{1}$, given that a particle is in the channel at time $t$.

The cross-sectional geometry of natural river channels can vary to a wide extent, and therefore also the velocity and diffusivity profiles can vary significantly. The analysis presented in Section 4 shows that the particular diffusivity and velocity profile have no major effect on the large-time behavior of the concentration distributions, because at large times the longitudinal spreading of the solute is controlled by the diffusivity profile in the porous medium and by the difference between the average velocity in the channel and the sediment bed. Since lateral velocity gradients typically dominate the dispersion process in the channel, it can be argued whether the anomalous behavior of the moments described in the previous sections for the case of vertical plane flow would still overcome the longitudinal dispersion produced by lateral velocity gradients in the 3D case. A sensitivity analysis was carried out for the crosssectional geometry in Figure 6, which represents a rounded rectangle with central depth $h$, base width $w$ and surface width $w_{s}=w+2 h$. Given the cylindrical symmetry of the geometry around the axes $O_{r}$ and $O_{l}$ in Figure 6, it is reasonable to assume cylindrical symmetry for the diffusivity profile around the same axes for $|y|>w / 2$, whereas for $|y| \leq w / 2$ the transverse diffusivity is a function of $z$ only. Accordingly, the diffusivity profile can be described by (33) provided that the coordinate $\hat{z}$ is replaced by the variable $\hat{z}_{r}$ such that

$$
\hat{z}_{r}= \begin{cases}\hat{z} & \text { for }|\hat{y}| \leq \hat{w} / 2 \\ 1-\sqrt{\left(|\hat{y}|-\frac{\hat{w}}{2}\right)^{2}+(\hat{z}-1)^{2}} & \text { for }|\hat{y}|>\hat{w} / 2\end{cases}
$$


where $\hat{y}=y / h$ and $\hat{w}=w / h$, and by replacing the normalization condition (12) with the fol-

lowing:

$$
\frac{h^{2}}{A} \iint_{A} \hat{\varepsilon}(\hat{y}, \hat{z}) d \hat{y} d \hat{z}=\frac{1}{\pi / 2+\hat{w}} \iint_{A} \hat{\varepsilon}(\hat{y}, \hat{z}) d \hat{y} d \hat{z}=1
$$

where the integral is calculated over the area $A$ of the channel cross-section. The analytical expressions for the coefficients $b_{i}$ and the parameter $\hat{\varepsilon}_{s}$ in (33) for the 3D case are given in Appendix A: (equations (A.17)-(A.21)).

Figure 6. Channel cross-sectional geometry considered in Section 5.

In reality, the cross-sectional profiles of the vertical and lateral diffusivity are different due to the anisotropic nature of turbulence, and a detailed analysis of the problem would require CFD simulation of the flow in the channel and the sediment bed. However, since the aim of the analysis presented here is to examine the general behavior of the concentration distributions and the scaling of their moments at times larger than $\tau_{1}$, a detailed CFD analysis is not necessary. As shown in Section 4, the behavior of the moments at times larger than $\tau_{1}$ obtained by assuming equation (33) instead of (13) for the diffusivity profile is virtually the same, thus equation (33) is assumed for the transverse diffusivity. This corresponds to assuming that the eddy diffusivity is constant at a distance $z_{m}$ from the channel boundaries.

The effect of the velocity gradients in the channel and the porous bed on longitudinal dispersion is analyzed assuming a plausible parameterization of the velocity profile that complies with the geometry of the cross-section. This approach is sufficient for the objective of the present study, which is to obtain general insights into the qualitative behavior of the moments of the concentration distributions, and the relative effect of $v(z)$ and $\varepsilon(z)$. A more detailed analysis would require that the properties of the flow are determined on a case-by-case basis using CFD simulation. With these considerations in mind, we can assume that the lateral velocity distribution in the channel is described by a beta distribution [Seo and Baek, 2004], whereas the vertical velocity profile is described by a power-law relationship. Consistently with the diffusivity profile, the velocity decreases exponentially in the sediment bed. Hence, the following distribution is assumed:

$$
\hat{v}(\hat{y}, \hat{z})= \begin{cases}\zeta_{u}\left(\frac{\hat{y}+\hat{w}_{z}(\hat{z}) / 2}{\hat{w}_{z}(\hat{z})}\right)^{\alpha_{u}}\left(1-\frac{\hat{y}+\hat{w}_{z}(\hat{z}) / 2}{\hat{w}_{z}(\hat{z})}\right)^{1-\beta_{u}} \hat{z}^{1 / n_{u}}+\hat{v}_{0} & \text { for } 0<\hat{z}_{r} \leq 1 \\ \left(\hat{v}_{0}-\hat{v}_{\infty}\right) e^{\hat{z}_{r} / \hat{h}_{u}+\hat{v}_{\infty}} & \text { for } \hat{z}_{r} \leq 0\end{cases}
$$


where $\hat{z}_{r} \equiv \hat{z}_{r}(\hat{y}, \hat{z})$ is given by (38), and

$$
\hat{w}_{z}(\hat{z})=2 \sqrt{1-(\hat{z}-1)^{2}}+\hat{w}
$$

The coefficient $\zeta_{u}$ must be determined by imposing the normalization condition:

$$
\frac{h^{2}}{A} \iint_{A} \hat{v}(\hat{y}, \hat{z}) d \hat{y} d \hat{z}=\frac{1}{\pi / 2+\hat{w}} \iint_{A} \hat{v}(\hat{y}, \hat{z}) d \hat{y} d \hat{z}=1
$$

where the integral is calculated over the area $A$ of the channel cross-section.

Consistently with the advection-diffusion equation (1), with $v$ and $\varepsilon$ now function of $y$ and $z$, the position of a solute particle is described by the following stochastic differential equations [Kitanidis, 1994]:

$$
\begin{aligned}
& d \hat{X}_{t}=\hat{v}\left(\hat{Y}_{t}, \hat{Z}_{t}\right) d \hat{t} \\
& d \hat{Y}_{t}=\left.\frac{d \hat{\varepsilon}}{d \hat{y}}\right|_{\hat{y}=\hat{Y}_{t}, \hat{z}=\hat{Z}_{t}} d \hat{t}+\sqrt{2 \hat{\varepsilon}\left(\hat{Y}_{t}, \hat{Z}_{t}\right) d \hat{t}} \eta_{y, t} \\
& d \hat{Z}_{t}=\left.\frac{d \hat{\varepsilon}}{d \hat{z}}\right|_{\hat{y}=\hat{Y}_{t}, \hat{z}=\hat{Z}_{t}} d \hat{t}+\sqrt{2 \hat{\varepsilon}\left(\hat{Y}_{t}, \hat{Z}_{t}\right) d \hat{t}} \eta_{z, t}
\end{aligned}
$$

where it was assumed $\varepsilon=\varepsilon_{y}=\varepsilon_{z}$, and the contribution to the longitudinal spreading induced by the microscale dispersion in the $x$-direction, $\varepsilon_{x}$, was neglected in line with the assumptions discussed in Section 4. Note that, in rivers, the vertical mixing coefficient, $\varepsilon_{z}$, is generally smaller than the lateral mixing coefficient, $\varepsilon_{y}$ [Fischer et al., 1979], but for a qualitative analysis of the moments, $m_{X}$, they are assumed to be the same so as to reduce the parametric space of the simulations. A generalization is straightforward. Equations (43)-(45) are solved using the weak order 2.0 predictor-corrector method described by Platen [1995] with time step $\Delta \hat{t}=$ $10^{-3}$. In all simulations, an instantaneous injection at $\hat{\mathbf{X}}=(0,0,1)$ is considered.

The parametric scenarios considered in the simulations are reported in Table 4. In Run E1 the surface width of the channel is $\hat{w}_{s}=20$, corresponding to a lateral mixing time scale $\tau_{1, w}=\hat{w}_{s}^{2} \tau_{1}=400 \tau_{1}$, whereas in Runs E-2 to E-7 the surface width is $\hat{w}=10$, and the lateral mixing time scale is $\tau_{1, w}=100 \tau_{1}$. Run E-3 is characterized by a large variation of the velocity throughout the cross-section, as opposed to all the other runs where the velocity gradients are confined within a narrow region near the boundaries of the cross-section. The values of $\alpha_{u}$ and $\beta_{u}$ in all runs except E-3 were calculated as $\alpha_{u}=\beta_{u}=1+1 / n_{u}$, which ensures that the near-bed vertical gradients in the central region, $|\hat{y}| \leq \hat{w} / 2$, are similar to the lateral gradients near the channel banks. Run E-5 has the same $\varepsilon_{0}$ and $h_{\infty}$ as Runs E-2 and E-3, but non-zero subsurface velocity (i.e. $\hat{v}_{0}$ and $\hat{v}_{\infty}>0$ ) and subsurface diffusivity $\hat{\varepsilon}_{\infty}$. 
Table 4. Parameters used in the random walk simulations of dispersion in a 3D channel.

\begin{tabular}{lccccccccccc}
\hline Run No. $\quad \hat{\varepsilon}_{0}$ & $\hat{\varepsilon}_{\infty}$ & $\hat{h}_{\infty}$ & $\hat{z}_{m}$ & $\hat{w}_{s}$ & $n_{u}$ & $\hat{v}_{0}$ & $\hat{v}_{\infty}$ & $\alpha_{u}$ & $\beta_{u}$ & $\hat{h}_{u}$ \\
\hline Run E-1 & $10^{-4}$ & 0 & 0.02 & 0.5 & 20 & 7 & 0 & 0 & 1.146 & 1.146 & - \\
Run E-2 & $10^{-3}$ & 0 & 0.02 & 0.5 & 10 & 7 & 0 & 0 & 1.146 & 1.146 & - \\
Run E-3 & $10^{-3}$ & 0 & 0.02 & 0.5 & 10 & 2 & 0 & 0 & 2 & 2 & - \\
Run E-4 & $10^{-4}$ & 0 & 0.02 & 0.2 & 10 & 7 & 0 & 0 & 1.146 & 1.146 & - \\
Run E-5 & $10^{-3}$ & $10^{-5}$ & 0.02 & 0.5 & 10 & 7 & $10^{-2}$ & $10^{-3}$ & 1.146 & 1.146 & 0.02 \\
Run E-6 & 0.1 & 0 & 0.3 & 0.5 & 10 & 7 & 0 & 0 & 1.146 & 1.146 & - \\
Run E-7 & 0.03 & 0 & 0.3 & 0.5 & 10 & 7 & 0 & 0 & 1.146 & 1.146 & - \\
\hline
\end{tabular}

Figure 7 shows the spatial moments derived for the parametric scenarios presented in Table 4. In the figure, the continuous lines represent the spatial moments and skewness obtained from the moments of $T_{1}$ according to (28), whereas the dashed lines represent the moments $m_{X, n}$ and skewness $\gamma_{X}$ resulting from (43). Same runs are represented by the same color. It is worth stressing that the moments of $T_{1}$ depend only on transverse mixing, and can therefore be deduced by solving equations (44) and (45) without consideration of (43).

The qualitative behavior of the moments found for the 3D channel confirms the results obtained for the two-dimensional case. It can be seen that, for times sufficiently larger than the lateral mixing time scale, $\tau_{1, w}=\hat{w}_{s}^{2} \tau_{1}$, the spatial moments $m_{X}$ converge to those deduced from $m_{T_{1}}$ under the assumption of uniform velocity in the two domains. Interestingly, the coefficient of skewness $\gamma_{X}$ in Run E-3 and E-4 is almost constant throughout the simulation period, whereas in Run E-3 $\gamma_{X}$ increases (i.e. becomes less negative) slightly in the temporal range between the vertical mixing time scale and the lateral mixing time scale, but then decreases (i.e. becomes more negative) at longer times. In this case the variance (Figure 7b) exhibits an intermediate range in which $m_{X, 2} \propto t^{3 / 2}$, whereas in Runs E-1, E-2, E-4 and E-5 $m_{X, 2} \propto t^{2}$. Intermediate scaling exponents between $3 / 2$ and 2 are observed for Runs E-6 and E-7, in which the interfacial diffusivity $\hat{\varepsilon}_{0}$ and attenuation depth $\hat{h}_{\infty}$ are significantly larger than in the other runs. It is also observed that, within the simulation time scale, there is no significant difference between the spatial moments in Run E-5 and the moments in Run E-2. This is expected because $\varepsilon_{\infty}$ is relatively small, and equation (28) implies that, if $u_{2} \ll u_{1}$, the error in the moments resulting from assuming $u_{2} \approx 0$ is small. 
Figure 7. Spatial moments $m_{X, n}, n=1,2$, and CSK for the parametric scenarios in Table 4. The dashed lines represent the curves obtained from particle tracking simulations of $X$ whereas the continuous lines are obtained from the moments of $T_{1}$ according to (28) using particle tracking simulations of transverse mixing only.

\section{Concentration Distribution in the Surface Water}

\subsection{Approximation at Large Times}

As described in Section 2, the PDF $\psi\left(x, t \mid \mathbf{x}_{0}\right)$ represents a normalized concentration distribution resulting from an instantaneous injection at point $\mathbf{x}_{0}$. In general, the distribution $\psi\left(x, t \mid \mathbf{x}_{0}\right)$ is linked to the conditional distribution of the residence time in the surface water, $f\left(t_{1}, t \mid z_{0}\right)$, by equation (4). This further simplifies to equation (7) if dispersion in the surface water and in the underlying porous medium are neglected.

The numerical simulations of transverse mixing presented in the previous section show that the conditional probability of the residence time in the surface water, $f\left(t_{1}, t \mid z_{0}\right)$ is well approximated by a beta distribution with time-dependent parameters, $a, \alpha$ and $\beta$. From equations (26) and (7) it follows that, for an instantaneous injection at point $\mathbf{x}_{0}=(0, h)$, the probability density $\psi$ can be expressed as

$$
\psi(x, t)=\frac{1}{\mathrm{~B}(\alpha, \beta) a u t}\left(\frac{x}{a u t}\right)^{\alpha-1}\left(1-\frac{x}{a u t}\right)^{\beta-1}
$$

where $u=u_{1}$ is the velocity in the main channel. The same equation applies to the more general case of an injection at point $\mathbf{x}_{0}=\left(x_{0}, h\right)$ and a constant velocity $u_{2}$ in the porous medium provided that $u=u_{1}-u_{2}$ and the variable $x$ is substituted with $x^{\prime}=x-x_{0}-u_{2} t$. If an injection is made at a vertical coordinate $z_{0} \neq h$, equation (46) may still be applicable, but the parameters $a, \alpha$ and $\beta$ will vary as a function of $z_{0}$.

The numerical particle tracking simulations show that, depending on the particular form of the diffusivity profile, the distributions can exhibit clear deviations from the Fickian behavior, with anomalous scaling of the first and second central moments, and persistent skewness over a broad range of temporal scales. In all the simulations presented in Section 3, the first and second central moments are well approximated by a power-law at long times. The coefficient of skewness can also be approximated by a power-law within suitable time ranges. This 
suggests the following asymptotic form for the parameters of the distribution (46):

$$
\begin{aligned}
& a \sim k_{a}\left(\frac{t}{\tau_{1}}\right)^{-e_{a}} \\
& \alpha \sim k_{\alpha}\left(\frac{t}{\tau_{1}}\right)^{e_{\alpha}} \\
& \beta \sim k_{\beta}\left(\frac{t}{\tau_{1}}\right)^{e_{\beta}}
\end{aligned}
$$

Under this assumption, it will be shown that the first three moments of the concentration dis-

tributions have power-law behavior, and Fickian dispersion is obtained as a special case.

\subsection{Spatial Moments}

For an instantaneous injection of a mass $M$ at point $\mathbf{x}_{0}$, the moments of the spatial distribution of the cross-sectionally averaged concentration in the surface water normalized by the area under the curve (equation 9), are equal to the moments of the PDF $\psi\left(x, t \mid \mathbf{x}_{0}\right)$. If the velocity is assumed to be uniform in each layer and longitudinal dispersion in each layer is neglected, then equation (6) holds, and hence the $n$-th central moment of $X$ (or, as mentioned above, the translated variable $X-x_{0}-u_{2} t$ if $x_{0}, u_{2} \neq 0$ ) for the particles in the surface water at time $t$ is given by (28). Equation (28) and (25) imply that the distributions $\psi(x, t)$ and $f\left(t_{1}, t\right)$ have the same skewness, $\gamma_{X}=\gamma_{T_{1}}$. Accordingly, the moments of the normalized concentration distributions for the numerical simulations presented in Section 3 follow similar curves to those in Figure 3, since the first and second central moments of $X$ are proportional to those of $T_{1}$ (Figure $3 \mathrm{~b}$ and $3 \mathrm{c}$, respectively), and the skewness is the same (Figure 3d). Having shown that the distribution (46) is a suitable approximation for $\psi(x, t)$, and that the moments of $X$ for the particles in the surface water exhibit the same anomalous scalings of the residence time $T_{1}$ in the surface water analyzed in Section 3, an asymptotic expression can now be derived for the moments of the distribution (46) under the assumption of asymptotic power-law behavior for the parameters $a, \alpha$ and $\beta$ (equations 47-49).

The first and second central moments of $\psi$ can be inferred from the moments of the beta distribution [Kokoska and Zwillinger, 2000]. These are, respectively,

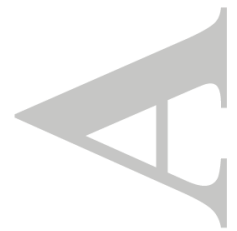

$$
\begin{aligned}
& m_{X, 1}=\frac{\alpha a u t}{\alpha+\beta} \\
& m_{X, 2}=\frac{\alpha \beta a^{2} u^{2} t^{2}}{(\alpha+\beta)^{2}(1+\alpha+\beta)}
\end{aligned}
$$

and the skewness is:

$$
\gamma_{X}=\frac{2(\beta-\alpha) \sqrt{1+\alpha+\beta}}{(2+\alpha+\beta) \sqrt{\alpha \beta}}
$$


In Appendix B: the moments and the CSK of $\psi$ are calculated considering also the effect of dispersion in the surface water, and are shown to converge to the expressions (50)-(52) at long times. The result implies that longitudinal dispersion at times sufficiently larger than the crosssectional mixing time scale of the channel is dominated by the spreading induced by the difference between the average velocity in the surface water and the average velocity in the sediment bed. This confirms the results of the sensitivity analysis presented in Section 4.

By substituting equations (47)-(49) for the parameters $a, \alpha$ and $\beta$ into (50)-(52), the asymptotic scaling relationships for the spatial moments at large times $\left(t \gg \tau_{1}\right)$ are found to be, for $e_{\alpha}>e_{\beta}$ :

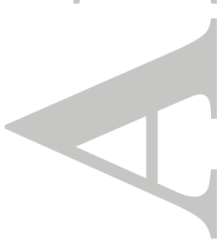

$$
\begin{aligned}
m_{X, 1} & \sim k_{a} u \tau_{1}\left(\frac{t}{\tau_{1}}\right)^{1-e_{a}} \\
m_{X, 2} & \sim \frac{k_{\beta} k_{a}^{2} u^{2} \tau_{1}^{2}}{k_{\alpha}^{2}}\left(\frac{t}{\tau_{1}}\right)^{2\left(1-e_{a}-e_{\alpha}\right)+e_{\beta}} \\
\gamma_{X} & \sim-\frac{2}{\sqrt{k_{\beta}}}\left(\frac{t}{\tau_{1}}\right)^{-e_{\beta} / 2}
\end{aligned}
$$

and, for $e_{\alpha}=e_{\beta}$ :

$$
\begin{aligned}
m_{X, 1} & \sim \frac{k_{\alpha} k_{a} u \tau_{1}}{k_{\alpha}+k_{\beta}}\left(\frac{t}{\tau_{1}}\right)^{1-e_{a}} \\
m_{X, 2} & \sim \frac{k_{\alpha} k_{\beta} k_{a}^{2} u^{2} \tau_{1}^{2}}{\left(k_{\alpha}+k_{\beta}\right)^{3}}\left(\frac{t}{\tau_{1}}\right)^{2\left(1-e_{a}\right)-e_{\alpha}} \\
\gamma_{X} & \sim-\frac{2\left(k_{\alpha}-k_{\beta}\right)}{\sqrt{\left(k_{\alpha}+k_{\beta}\right) k_{\alpha} k_{\beta}}}\left(\frac{t}{\tau_{1}}\right)^{-e_{\alpha} / 2}
\end{aligned}
$$

The case of $e_{\alpha}<e_{\beta}$ is not considered as the resulting spatial distributions would have positive skewness and decreasing mean, which does not appear to be representative of any realistic scenario.

As a special case, if $e_{a}=0$ (i.e. constant $a$ ), $e_{\alpha}=e_{\beta}=1$ and $k_{\alpha}=k_{\beta}$, the scaling relationships (56)-(58) are consistent with a Fickian diffusion process, because the first and the second central moments increase linearly with time and the spatial skewness is zero. Under these assumptions, equations (56)-(58) imply that the equivalent velocity and longitudinal dispersion coefficient are, respectively,

$$
\begin{aligned}
& \bar{u}=\frac{m_{X, 1}}{t}=\frac{k_{\alpha} k_{a} u}{k_{\alpha}+k_{\beta}}=\frac{a u}{2} \\
& \bar{D}=\frac{m_{X, 2}}{2 t}=\frac{1}{2} \frac{k_{\alpha} k_{\beta} k_{a}^{2} u^{2} \tau_{1}}{\left(k_{\alpha}+k_{\beta}\right)^{3}}=\frac{a^{2} u^{2} \tau_{1}}{16 k_{\alpha}}
\end{aligned}
$$

where it was considered that $k_{\alpha}=k_{\beta}$ and $a=k_{a}$. 


\subsection{Temporal Moments}

The behavior of the temporal moments of the breakthrough curves in the surface wa-

ter is analyzed using the analytical approximation of the conditional density $f\left(t_{1}, t \mid z_{0}\right)$ expressed by equation (26) for its dimensionless counterpart $f^{*}$. An approximate expression for the moments is derived by assuming that the parameters $a, \alpha$ and $\beta$ vary slowly over time, so that the temporal evolution of the concentration at a section generated by the passage of the solute plume can be inferred from (26) assuming constant parameters. The numerical simulations presented in Section 3 suggest that this assumption is applicable at large times.

The raw temporal moment of order $n$ is defined as:

$$
\mu_{T, n}=\int_{0}^{\infty} t^{n} \psi(x, t) d t
$$

and the normalized temporal moment of order $n$ is:

$$
m_{T, n}^{\prime}=\frac{\mu_{T, n}}{\mu_{T, 0}}
$$

from which the first three central moments can be obtained as follows [Kenney and Keeping,

1947; Pannone, 2012]:

$$
\begin{aligned}
& m_{T, 2}=m_{T, 2}^{\prime}-\left(m_{T, 1}\right)^{2} \\
& m_{T, 3}=m_{T, 3}^{\prime}-3 m_{T, 1} m_{T, 2}^{\prime}+2 m_{T, 1}^{3}
\end{aligned}
$$

where $m_{T, 1}=m_{T, 1}^{\prime}$. The existence of the $n$-th order moment requires that $\alpha>n+1, n=$ $1,2, \ldots$ Consistently with equations (47)-(48) with $e_{\alpha}>0$ and $k_{\alpha}>0$, this condition is satisfied at sufficiently long times for any finite $n$.

Calculation of the integral (61), taking $a, \alpha$ and $\beta$ as constant, yields the following general expression for the $n$-th raw moment:

$$
\mu_{T, n}=\frac{\mathrm{B}(\alpha-1-n, \beta) x^{n}}{\mathrm{~B}(\alpha, \beta)(a u)^{n+1}}
$$

hence, from (62), the moments of the normalized temporal distributions are:

$$
m_{T, n}^{\prime}=\frac{\mathrm{B}(\alpha-1-n, \beta)}{\mathrm{B}(\alpha-1, \beta)}\left(\frac{x}{a u}\right)^{n}
$$

and therefore, from (62)-(65),

$$
\begin{aligned}
m_{T, 1} & =\frac{(\alpha+\beta-2) x}{(\alpha-2) a u} \\
m_{T, 2} & =\frac{\beta(\alpha+\beta-2) x^{2}}{(\alpha-3)(\alpha-2)^{2} a^{2} u^{2}} \\
m_{T, 3} & =\frac{2 \beta(\alpha+\beta-2)(\alpha+2 \beta-2) x^{3}}{(\alpha-4)(\alpha-3)(\alpha-2)^{3} a^{3} u^{3}}
\end{aligned}
$$


Accordingly, the skewness is given by:

$$
\gamma_{T}=\frac{2(\alpha+2 \beta-2) \sqrt{\alpha-3}}{(\alpha-4) \sqrt{\beta(\alpha+\beta-2)}}
$$

In Appendix C: the moments of $\psi$ are calculated considering also the effect of dispersion in the surface water, and are shown to converge to the expressions (67)-(70) at large times.

It must be stressed that in the equations above, the parameters $a, \alpha$ and $\beta$ are functions of time, and therefore the scaling exponent of the temporal moments cannot be inferred trivially from the exponent of the variable $x$ that appears explicitly in the equations. The asymptotic expressions for the moments $m_{T, 1}$ and $m_{T, 2}$ and the skewness coefficient $\gamma_{T}$ are found by approximating the parameters $a, \alpha$ and $\beta$ in (67)-(70) with the asymptotic relationships (47)-(49) calculated at the time when the centroid of the spatial distribution passes through section $x$, i.e. $m_{X, 1}=x$. For $e_{\alpha}>e_{\beta}$, equation (53) gives:

$$
t=\tau_{1}\left(\frac{x}{k_{a} u \tau_{1}}\right)^{\frac{1}{1-e_{a}}}
$$

whereas for $e_{\alpha}=e_{\beta}$, equation (56) provides:

$$
t=\tau_{1}\left[\frac{\left(k_{\alpha}+k_{\beta}\right) x}{k_{\alpha} k_{a} u \tau_{1}}\right]^{\frac{1}{1-e_{a}}}
$$

$$
\begin{aligned}
\text { Hence, for } e_{\alpha}>e_{\beta} \text { : } & \\
m_{T, 1} & \sim \tau_{1}\left(\frac{x}{k_{a} u \tau_{1}}\right)^{\frac{1}{1-e_{a}}} \\
m_{T, 2} & \sim \frac{k_{\beta} \tau_{1}^{2}}{k_{\alpha}^{2}}\left(\frac{x}{k_{a} u \tau_{1}}\right)^{\frac{2-2 e_{\alpha}+e_{\beta}}{1-e_{a}}} \\
\gamma_{T} & \sim \frac{2}{\sqrt{k_{\beta}}}\left(\frac{x}{k_{a} u \tau_{1}}\right)^{-\frac{e_{\beta}}{2\left(1-e_{a}\right)}}
\end{aligned}
$$

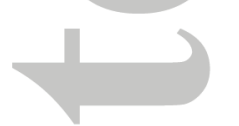

and, for $e_{\alpha}=e_{\beta}$ :

$$
\begin{aligned}
m_{T, 1} & \sim \tau_{1}\left[\frac{\left(k_{\alpha}+k_{\beta}\right) x}{k_{\alpha} k_{a} u \tau_{1}}\right]^{\frac{1}{1-e_{a}}} \\
m_{T, 2} & \sim \frac{k_{\beta} \tau_{1}^{2}}{k_{\alpha}\left(k_{\alpha}+k_{\beta}\right)}\left[\frac{\left(k_{\alpha}+k_{\beta}\right) x}{k_{\alpha} k_{a} u \tau_{1}}\right]^{\frac{2-e_{\alpha}}{1-e_{a}}} \\
\gamma_{T} & \sim \frac{2\left(k_{\alpha}+2 k_{\beta}\right)}{\sqrt{k_{\alpha} k_{\beta}\left(k_{\alpha}+k_{\beta}\right)}}\left[\frac{\left(k_{\alpha}+k_{\beta}\right) x}{k_{\alpha} k_{a} u \tau_{1}}\right]^{-\frac{e_{\alpha}}{2\left(1-e_{a}\right)}}
\end{aligned}
$$

When $e_{\beta}=0, \beta$ and $\gamma_{T}$ are constant at long times. If $e_{\alpha}=0$, then also $\alpha$ is constant,

and the resulting scaling of the first and second central moments is $m_{T, 1} \propto x$ and $m_{T, 2} \propto x^{2}$, respectively. If $e_{\alpha}>0$, then the temporal and spatial skewness at long times is

$$
\gamma_{X}=\gamma_{T}=\frac{2}{\sqrt{\beta}}
$$


In particular, for $\gamma_{T} \approx 1.2$, as reported by González-Pinzón et al. [2013], $\beta \approx 2.8$. If $e_{a}=0$

then the first spatial and temporal moments are linear in $t$ and $x$, respectively, whereas the scaling of the second central moment is controlled by $e_{\alpha}$. The experimental results of Nordin and Troutman [1980], in which the variance scales linearly with distance, correspond to $e_{\alpha}=1 / 2$, whereas the results of the meta-analysis of González-Pinzón et al. [2013], suggesting $m_{T, 2} \propto$ $t^{1.568}$, correspond to $e_{\alpha}=0.216$.

The validity of the expression derived above under the frozen cloud approximation is confirmed by numerical simulations. Figure 8 shows the behavior of the mean, variance and skewness of the normalized breakthrough curves generated numerically from equation (46) using

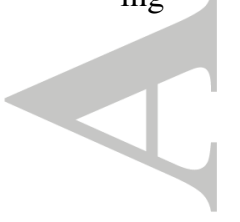

$$
\begin{aligned}
& a=k_{a}\left(1+t / \tau_{1}\right)^{-e_{a}} \\
& \alpha=\alpha_{0}+k_{\alpha}\left(t / \tau_{1}\right)^{e_{\alpha}} \\
& \beta=\beta_{0}+k_{\beta}\left(t / \tau_{1}\right)^{e_{\beta}}
\end{aligned}
$$

with the parameters reported in Table 5. Equations (80)-(82) are consistent with the asymptotic relationships (47)-(49) at long times. In the figure, the distance is normalized by the mixing length $\ell_{1}=u h_{1}^{2} / \varepsilon_{1}$. In all cases, the scaling of the centroid is asymptotically linear, whereas the scaling of the variance is linear for Run B-1 and B-2 and super-diffusive for the other runs, with $m_{T, 2} \propto t^{3 / 2}$ for Run B-2 and $m_{T, 2} \propto t^{2}$ for Run B-4. In Run B-1 the skewness (Figure 8c) approaches zero at long times, as expected in case of Fickian dispersion, whereas in Run B-4 the skewness is constant. In Runs B-2 and B-3 the skewness approaches a constant non-zero value, $\gamma_{T}=1.2$. The normalized temporal distributions $\varphi(x, t)=\psi(x, t) / \mu_{T, 0}$ generated according to (46) for $x / \ell_{1}=30,300,3000$ and $3 \times 10^{4}$ are plotted in non-dimensional form in Figure 9 for Runs B-1, B-2 and B-3.

Figure 8. Temporal moments as a function of the distance from the injection point generated numerically from equation (46) under the asymptotic power-law assumption for $a, \alpha$ and $\beta$ (80-82) with the parameters reported in Table 5.

Finally, it must be proved whether the reduction of mass in the surface water expressed by the factor $p(t)$ in equation (9) would cause the normalized concentration distributions to deviate from the scaling relationships found above for the temporal moments of $\psi(x, t)$. The 
Table 5. Coefficients and exponents of equations (80), (81) and (82) used for the parameters $a, \alpha$ and $\beta$ in equation (46) in the numerical simulations of the temporal moments presented in Figure 8 and the breakthrough curves in Figure 9.

\begin{tabular}{ccccccccc}
\hline Run No. & $\alpha_{0}$ & $\beta_{0}$ & $k_{a}$ & $k_{\alpha}$ & $k_{\beta}$ & $e_{a}$ & $e_{\alpha}$ & $e_{\beta}$ \\
\hline Run B-1 & 1 & 1 & 1 & 0.1 & 0.1 & 0 & 1 & 1 \\
Run B-2 & 3 & 0 & 0.5 & 10 & 2.8 & 0 & $1 / 4$ & 0 \\
Run B-3 & 3 & 0 & 0.5 & 0.85 & 2.8 & 0 & $1 / 2$ & 0 \\
Run B-4 & 0 & 0 & 0.75 & 8 & 2.8 & 0 & 0 & 0 \\
\hline
\end{tabular}

Figure 9. Normalized breakthrough curves, $\varphi(x, t)=\psi(x, t) / \mu_{T, 0}$, generated by equation (46) under the asymptotic power-law assumption for $a, \alpha$ and $\beta$ (80-82) with the parameters in Table 5 for different longitudinal distances from the injection point. In the graphs, time is normalized by the time scale of vertical mixing in the surface water (upper layer), $\tau_{1}$, whereas distance is normalized by the mixing length scale $\ell_{1}=u_{1} \tau_{1}$.

raw moments of the concentration distributions can be derived from equation (9) as follows:

$$
\mu_{T, n}^{(c)}=\int_{0}^{\infty} t^{n} c(x, t) d t=\frac{M}{A} \int_{0}^{\infty} t^{n} p(t) \psi(x, t) d t
$$

If the function $p(t)$ varies slowly during the passage of the solute plume through a section, then the factor $p(t)$ can be taken out of the integral (9), thus the moments of the normalized concentration distributions are the same as the normalized function $\psi$. This assumption can be further relaxed to a requirement that $p(t)$ can be locally approximated by a power law, $p(t) \propto$ $t^{-e_{p}}$, where $e_{p}$ is a non-negative exponent. The resulting moments of the normalized concentration distribution become:

$$
m_{T, n}^{\prime(c)}=\frac{\mathrm{B}\left(\alpha+e_{p}-1-n, \beta\right)}{\mathrm{B}\left(\alpha+e_{p}-1, \beta\right)}\left(\frac{x}{a u}\right)^{n}
$$

Since the variable $x$ and the time-dependent parameters $a, \alpha$ and $\beta$ appear in (84) with the same exponents as in (66), the resulting scaling relationships deduced from the concentration distributions would be the same as (73)-(75). The assumption that $p(t)$ can be well approximated piecewise by a power law within increasingly longer time intervals is supported by the numerical results presented in Figure 3a. 


\section{Discussion}

The analysis presented in the previous sections shows that the anomalous scaling of the moments of the concentration distributions is a consequence of the incomplete transverse mixing in the porous medium, which produces a deviation from the conditions that allow for the one-dimensional reduction of the multi-dimensional advection-diffusion equation [Taylor, 1953].

This result applies not only to semi-infinite sediment beds, but also to finite beds as long as the time of observation of the concentration distributions is smaller than the time scale of mixing in the porous medium. Simulations show that the longitudinal dispersion of a solute in an open-channel flow over a porous bed in which the transverse dispersivity decreases exponentially with depth cannot be approximated as a Fickian dispersion process, even though the dispersivity is practically zero at a certain depth. The results obtained from the $2 \mathrm{D}$ analysis of vertical and longitudinal mixing show that, for relatively small attenuation depths $\hat{h}_{\infty}$, the variance of the concentration distributions scales as $t^{2}$. However, for greater interfacial diffusivities and attenuation depths (Runs A-2 and A-3 in the 2D case, and Runs E-6 and E-7 for the 3D case), and for larger variations of the cross-sectional velocity profile in a 3D channel (Run E-3), the simulations show intermediate scaling exponents between 1.5 and 2 . This is in agreement with the results of González-Pinzón et al. [2013] indicating a scaling exponent for the temporal variance $\approx 1.57$. For several of the parametric scenarios considered, both in the $2 \mathrm{D}$ and the $3 \mathrm{D}$ case, the coefficient of skewness was found to be approximately constant within a broad temporal range. This is, again, in agreement with the findings of González-Pinzón et al. [2013], and also Nordin and Troutman [1980], although in the simulations the value of CSK was found to vary within a broader range than suggested by experimental data. This might be due to particular parametric scenarios being more common in real streams and rivers, and to truncation errors in the experimental observations which reduce the skewness of tracer BTCs.

In representing the transport of a solute along the channel as a one-dimensional process, the evolution of the normalized cross-sectionally averaged solute concentration in the surface water was described by the probability density $\psi\left(x, t \mid z_{0}\right)$, which depends on the vertical coordinate of the injection point, $z_{0}$. This implies that, in contrast with classic one-dimensional formulations of solute transport, the process has memory of the initial vertical concentration profile, and therefore the function $\psi\left(x, t \mid z_{0}\right)$ cannot be used as a propagator to derive the evolution of the concentration along the channel unless the entire concentration distribution in the $x$ - and $z$-direction is known in the initial state. 
Because the distribution of the residence time in the surface water $f\left(t_{1}, t\right)$ can only take non-zero values in the finite interval $0 \leq t_{1} \leq t$, the spatial concentration distributions have finite moments. However, the moments of the breakthrough curves are not finite in general. Previous works have shown that the residence time distributions in the sediment resulting from interfacial diffusion with a semi-infinite porous medium have power-law tails that scale as $t^{-3 / 2}$ [Bottacin-Busolin and Marion, 2010]. The result is confirmed also by the work of Aquino et al. [2015] who derived an asymptotic relation between the scaling of the concentration peaks and the tail scaling of the breakthrough curves as a function of the downstream distance from the injection point. By performing particle tracking simulations for a two-layer flow in which the transverse turbulent dispersion coefficient is parabolic in the surface water and is constant in the sediment bed, they showed that the tails of the breakthrough curves at different sections downstream of the injection point scale as $t^{-3 / 2}$. The result implies that the moments of the BTCs of any order above zero do not exist. This might seem in contradiction with the analysis of the temporal moments presented in Section 6, in which the moments are finite. It must be noted, however, that the results presented in Section 6 are obtained under the approximation (46), which is representative of the bulk behavior of $\psi\left(x, t \mid z_{0}\right)$ but is not an accurate representation of the late-time tail behavior. As can been seen in Figure 2b, the approximation for the conditional density of the residence time in the surface water $f\left(t_{1}, t\right)$ expressed by equation (26) has a left tail that decreases faster than the distributions derived from the particle tracking simulations, which implies that the tail of the breakthrough curves (BTCs) generated under the approximation (46) decreases faster than the real BTCs. The temporal moments derived in Section 6.3 are therefore the moments of the distribution (46) that approximates the "bulk" behavior of the real distribution.

Even though, theoretically, the temporal moments are not finite for a semi-infinite bed, the moments of tracer test BTCs are always finite due to the finite duration of the observation period. The tail of the experimental distributions is also affected by detection limits of the instrument, background noise and experimental errors, which inevitably produce a truncation of the tail [Drummond et al., 2012]. The results of this work show that the signature of the exchange with the sediment bed lies not only in the long-tail behavior of the breakthrough curves, but also in the way that the moments of the BTCs scale with the distance from the injection point. Hence, if the parameters of the approximating distribution (46) can be linked to the profile of the transverse dispersion coefficient in the sediment, then the properties of the 
exchange with the bed can potentially be inferred by fitting the distribution (46) to the normalized BTCs.

Chatwin et al. [1995] used the beta distribution as an approximation for the probability density function of the concentration of a dispersant at a point in a turbulent flow field. Although the problem investigated by Chatwin et al. [1995] is different from the problem analyzed in this study, in both cases the variance of the distribution has super-diffusive behavior and the skewness is persistent, with no indication of a tendency to approach normality. The beta distribution was also found to describe the non-reactive solute concentration in heterogeneous aquifers under the hypothesis of statistical stationarity [Bellin and Tonina, 2007; Boso et al., 2013]. The introduction of the additional scale parameter $a$ in equation (26) and (46) allows to control the interval in which the residence time probability in the surface water is significantly different from zero. In this regard, it must be noted that in equation (46) the parameter $a$ is always multiplied by the velocity $u$, therefore the product $a u$ represents an apparent velocity, which in the general case can depend on time. It remains to be clarified how the parameters $\alpha, \beta$ and $a$ can be expressed as a function of time and space in response to the vertical profile of the transverse diffusivity.

The assumption that the parameters follow a power law at late times leads to power-law scaling of the mean and variance of the BTCs, which is consistent with the observations of several tracer tests [Day, 1975; Nordin and Sabol, 1974; Nordin and Troutman, 1980]. The asymptotic analysis presented in Section 6.3 shows that if the parameters have power-law scaling at late times, also the coefficient of skewness has power-law scaling at late times, with the case of a constant skewness as a special case. However, for the diffusivity profiles assumed in this study, Figure 3, 4 and 7 suggest that the behavior of the skewness cannot be fully represented by a power-law. This suggests that the CSK may be assumed to be constant [González-Pinzón et al., 2013] only as an approximation of scattered data observed in tracer tests.

Since the profile of the dispersion coefficient in the porous medium depends on the conductivity and the porosity of the porous medium, the structure of the sediment bed is likely to play an important role on the late-time behavior of the moments of the BTCs. Aubeneau et al. [2014] investigated the effect of substrate size and heterogeneity on anomalous transport in shallow streams, and found that the truncation times of the power-law tails of the BTCs depends on the composition and structure of the porous medium. The presence of irregularities of the bed surface, such as bedforms, can alter the behavior of the BTCs by inducing ad- 
vective flows in the sediment which enhance mixing [Marion et al., 2008b; Bottacin-Busolin and Marion, 2010; Boano et al., 2014], but the results of this work imply that the anomalous behavior of the moments at late times is controlled by diffusive processes in the hyporeic layer rather than advective processes.

\section{Conclusion}

The transport of a passive solute in open-channel flow over a semi-infinite flat porous bed was investigated using particle tracking simulations of advection and diffusion in a 2D and 3D channel. The simulations show that the moments of the concentration distributions in the channel have superdiffusive scaling and persistent skewness at times significantly larger than the cross-sectional mixing time scale of the channel. This result agrees with previous experimental findings indicating that the variance of tracer breakthrough curves is superlinear, and the skewness does not decrease with the distance from the injection point [Day, 1975; Nordin and Troutman, 1980; González-Pinzón et al., 2013]. A stochastic interpretation of the transport process along the channel was proposed that decouples longitudinal mixing in the channel and the porous bed from the transverse mixing process across the two domains. This decomposition shows that the late-time behavior of the moments of the solute concentration is controlled by the cross-sectional profile of the transverse mixing coefficient, and by the difference between the average velocity in the channel and in the porous bed. The results are confirmed by a sensitivity analysis of the effect of the velocity profile, which also shows that the transverse diffusivity profile in the surface water has a negligible effect on the behavior of the moments at long times. Instead, the main driver for the anomalous scaling of the moments at large times was found to be the profile of the in-bed transverse mixing coefficient, which in this study was assumed to be exponentially attenuated with the depth below the sediment-water interface in accordance with the experimental results of Chandler et al. [2016].

As pointed out by González-Pinzón et al. [2013], the non-Fickian scaling of the moments found in tracer tests data, as well as in the present study, is structurally inconsistent with 1D solute transport models traditionally used in tracer studies and catchment scale biogeochemical models. Simulation results suggest that a general form approximating the normalized concentration distributions in the surface water is given by the beta distribution with time dependent parameters. It was further shown that if the parameters of the approximating beta distribution are assumed to follow a power law at long times, the resulting spatial and temporal moments also follow a power law at long times. Asymptotic relationships were derived showing 
that a variety of scaling behaviors can be represented by this functional form, including Fickian dispersion as a special case. It was also shown that, under reasonable assumptions on the variation of the solute mass in the surface water, the continuous transfer of solute from the water in the channel to the sediment bed does not affect the scaling of the moments of the breakthrough curves in the surface water.

The results of this study have implications for direct and inverse modelling of transport processes in river channels. Since the 1-D advection-dispersion equation, often used in water quality models for rivers and river networks, is structurally inconsistent with the anomalous scaling of the moments of the concentration distributions observed in tracer tests, an alternative formulation is needed. In the present work, a functional form was proposed that approximates the concentration distribution resulting from an instantaneous point injection of solute in the surface water. Future studies should investigate the link between the parameters of the surface concentration distribution and the cross-sectional profile of the transverse mixing coefficient in the porous medium. This would allow to predictively evaluate the evolution of the concentration of a solute in the surface water if the properties of the porous medium are known, or alternatively to estimate the properties of the porous medium from tracer tests using inverse modelling approaches.

\section{[1}

\section{A: Polynomial Coefficients for Diffusivity Profile in Surface Water}

The coefficients $b_{i}$ in equation (13) resulting from the conditions (14)-(18) and (12) are given by:

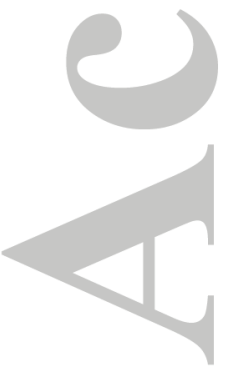

$$
\begin{aligned}
& b_{5}=4\left[\hat{\varepsilon}_{0}^{\prime}+\hat{\varepsilon}_{s}^{\prime}+6\left(\hat{\varepsilon}_{0}-\hat{\varepsilon}_{s}\right)\right] \\
& b_{4}=\frac{5}{2}\left(12-5 \hat{\varepsilon}_{0}^{\prime}-3 \hat{\varepsilon}_{s}^{\prime}-30 \hat{\varepsilon}_{0}+18 \hat{\varepsilon}_{s}\right) \\
& b_{3}=2\left(-30+7 \hat{\varepsilon}_{0}^{\prime}+2 \hat{\varepsilon}_{s}^{\prime}+40 \hat{\varepsilon}_{0}-10 \hat{\varepsilon}_{s}\right) \\
& b_{2}=\frac{1}{2}\left(60-13 \hat{\varepsilon}_{0}^{\prime}-\hat{\varepsilon}_{s}^{\prime}-60 \hat{\varepsilon}_{0}\right) \\
& b_{1}=\hat{\varepsilon}_{0}^{\prime} \\
& b_{0}=\hat{\varepsilon}_{0}
\end{aligned}
$$




$$
\begin{aligned}
& \hat{\varepsilon}_{s}=\hat{\varepsilon}(\hat{z}=1) \\
& \hat{\varepsilon}_{0}^{\prime}=\left.\frac{d \hat{\varepsilon}_{0}}{d \hat{z}}\right|_{\hat{z}=0} \\
& \hat{\varepsilon}_{s}^{\prime}=\left.\frac{d \hat{\varepsilon}_{0}}{d \hat{z}}\right|_{\hat{z}=1}
\end{aligned}
$$

In the 2D case, the coefficients $b_{i}$ and the parameter $\hat{\varepsilon}_{s}$ in (33) that satisfy conditions (34)-(37) and (12) are given by:

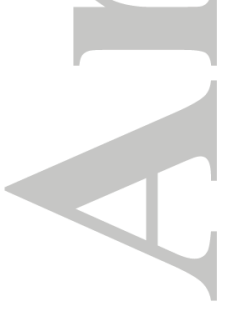

$$
\begin{aligned}
& b_{3}=\frac{2\left(6-6 \hat{\varepsilon}_{0}-3 \hat{\varepsilon}_{0}^{\prime} \hat{z}_{m}+\hat{\varepsilon}_{0}^{\prime} \hat{z}_{m}^{2}\right)}{3\left(\hat{z}_{m}-2\right) \hat{z}_{m}^{3}} \\
& b_{2}=\frac{-12+12 \hat{\varepsilon}_{0}+8 \hat{\varepsilon}_{0}^{\prime} \hat{z}_{m}-3 \hat{\varepsilon}_{0}^{\prime} \hat{z}_{m}^{2}}{2\left(\hat{z}_{m}-2\right) \hat{z}_{m}^{2}} \\
& b_{1}=\hat{\varepsilon}_{0}^{\prime} \\
& b_{0}=\hat{\varepsilon}_{0} \\
& \hat{\varepsilon}_{s}=\frac{-12+6 \hat{\varepsilon}_{0} \hat{z}_{m}+\hat{\varepsilon}_{0}^{\prime} \hat{z}_{m}^{2}}{6\left(\hat{z}_{m}-2\right)}
\end{aligned}
$$

For the 3D channel described in Section 5, the coefficients $b_{i}$ and the parameter $\hat{\varepsilon}_{s}$ in equation (33) that satisfy conditions (34)-(37), with $\hat{z}$ replaced by $\hat{z}_{r}$ (equation (38)), and (42) are given by:

$$
\begin{aligned}
& b_{3}=-\frac{5\left(-24 \hat{w}+24 \hat{w} \hat{\varepsilon}_{0}-12 \pi+12 \pi \hat{\varepsilon}_{0}+12 \hat{w} \hat{z}_{m} \hat{\varepsilon}_{0}^{\prime}+6 \pi \hat{z}_{m} \hat{\varepsilon}_{0}^{\prime}-4 \hat{w} \hat{z}_{m}^{2} \hat{\varepsilon}_{0}^{\prime}-\pi \hat{z}_{m}^{3} \hat{\varepsilon}_{0}^{\prime}\right)}{3 \hat{z}_{m}^{3}\left(-20 \hat{w}-10 \pi+10 \hat{w} \hat{z}_{m}+3 \pi \hat{z}_{m}^{2}\right)} \\
& b_{2}=-\frac{60 \hat{w}-60 \hat{w} \hat{\varepsilon}_{0}+30 \pi-30 \pi \hat{\varepsilon}_{0}-40 \hat{\varepsilon}_{0}^{\prime} \hat{w} \hat{z}_{m}-20 \pi \hat{z}_{m} \hat{\varepsilon}_{0}^{\prime}+15 \hat{w} \hat{z}_{m}^{2} \hat{\varepsilon}_{0}^{\prime}+4 \pi \hat{z}_{m}^{3} \hat{\varepsilon}_{0}^{\prime}}{\hat{z}_{m}^{2}\left(-20 \hat{w}-10 \pi+10 \hat{w} \hat{z}_{m}+3 \pi \hat{z}_{m}^{2}\right)} \text { (A.18) } \\
& b_{1}=\hat{\varepsilon}_{0}^{\prime} \\
& b_{0}=\hat{\varepsilon}_{0} \\
& \hat{\varepsilon}_{s}=-\frac{60 \hat{w}+30 \pi-30 \hat{w} \hat{\varepsilon}_{0} \hat{z}_{m}-5 \hat{\varepsilon}_{0}^{\prime} \hat{w} \hat{z}_{m}^{2}-9 \pi \hat{\varepsilon}_{0} \hat{z}_{m}^{2}-2 \pi \hat{\varepsilon}_{0}^{\prime} \hat{z}_{m}^{3}}{3\left(-20 \hat{w}-10 \pi+10 \hat{w} \hat{z}_{m}+3 \pi \hat{z}_{m}^{2}\right)}
\end{aligned}
$$

\section{B: Spatial Moments Considering In-channel Dispersion}

In this section, the spatial moments of the PDF $\psi(x, t)$ are derived considering the contribution of longitudinal dispersion in the surface water, while neglecting longitudinal dispersion in the porous bed where the velocity gradients are relatively smaller. To simplify the expressions, we consider the case in which $u=u_{1}, u_{2}=0$, and the solute is injected at $x_{0}=$ 0 . This corresponds to the assumption that the distribution of particle jumps in the surface water $g_{1}(x \mid t)$ is given by (5) and $g_{2}(x \mid t)=\delta(x)$. Using equation (4), the characteristic function 
of the distribution $\psi(x, t)$ is:

$$
\begin{aligned}
\Psi(\omega, t) & =\int_{-\infty}^{\infty} e^{i \omega x} \psi(x, t) d x \\
& =\int_{-\infty}^{\infty} e^{i \omega x} \int_{0}^{\infty} g_{1}\left(x \mid t_{1}\right) f\left(t_{1}, t\right) d t_{1} d x \\
& =\int_{0}^{\infty} \int_{-\infty}^{\infty} e^{i \omega x} g_{1}\left(x \mid t_{1}\right) d x f\left(t_{1}, t\right) d t_{1} \\
& =\int_{0}^{\infty} G_{1}\left(\omega \mid t_{1}\right) f\left(t_{1}, t\right) d t_{1}
\end{aligned}
$$

where $i$ is the imaginary unit, and

$$
\begin{aligned}
G_{1}\left(\omega \mid t_{1}\right) & =\int_{-\infty}^{\infty} e^{i \omega x} g_{1}\left(x \mid t_{1}\right) d x \\
& =\int_{-\infty}^{\infty} \frac{1}{2 \sqrt{\pi D_{1} t_{1}}} \exp \left[-\frac{\left(x-u t_{1}\right)^{2}}{4 D_{1} t_{1}}+i \omega x\right] d x \\
& =e^{\omega\left(i u-D_{1} \omega\right) t_{1}}
\end{aligned}
$$

Assuming that, consistently with (26), the dimensional PDF of the residence time in the surface water given $Z>0$ is

$$
f\left(t_{1}, t\right)=\frac{1}{a t \mathrm{~B}(\alpha, \beta)}\left(\frac{t_{1}}{a t}\right)^{\alpha-1}\left(1-\frac{t_{1}}{a t}\right)^{\beta-1}
$$

calculation of the integral (B.1) gives

$$
\begin{aligned}
\Psi(\omega, t) & ={ }_{1} F_{1}\left(\alpha, \alpha+\beta, a \omega\left(i u-D_{1} \omega\right) t\right) \\
& =\sum_{n=0}^{\infty} \frac{(\alpha)_{n}}{(\alpha+\beta)_{n}} \frac{\left[a \omega\left(i u-D_{1} \omega\right) t\right]^{n}}{n !}
\end{aligned}
$$

where ${ }_{1} F_{1}$ is Kummer's confluent hypergeometric function, and $(\alpha)_{n}=\alpha(\alpha+1) \cdots(\alpha+$ $n-1)$ is the rising factorial. The $n$-th order moment of $X$ is given by

$$
m_{X, n}=\left.(-i)^{n} \frac{d \Psi}{d \omega^{n}}\right|_{\omega=0}
$$

hence

$$
\begin{aligned}
& m_{X, 1}=\frac{a \alpha u t}{\alpha+\beta} \\
& m_{X, 2}=\frac{\alpha \beta a^{2} u^{2} t^{2}}{(\alpha+\beta)^{2}(1+\alpha+\beta)}+\frac{2 a \alpha D_{1} t}{\alpha+\beta}
\end{aligned}
$$

$$
\gamma_{X}=\frac{\left[2 \beta u^{3}(\beta-\alpha)(a t)^{3 / 2}+6 a \beta D_{1} u\left(\alpha^{2}+2 \alpha(\beta+1)+\beta(\beta+2)\right) \sqrt{a t}\right] \sqrt{1+\alpha+\beta}}{(\alpha+\beta+2)\left[a \beta u^{2} t+2 D_{1}\left(\alpha^{2}+2 \alpha \beta+\alpha+\beta^{2}+\beta\right)\right]^{3 / 2} \sqrt{\alpha}}
$$

At long times equations (B.6), (B.7) and (B.8) converge to (50), (51) and (52), respectively. 


\section{C: Temporal Moments Considering In-channel Dispersion}

Under the same assumptions presented in Appendix B:, the temporal moments of the PDF $\psi(x, t)$ are derived as follows. The Laplace transform of $\psi(x, t)$ is:

$$
\begin{aligned}
\tilde{\psi}(x, s) & =\int_{0}^{\infty} e^{-s t} \psi(x, t) d t \\
& =\int_{0}^{\infty} e^{-s t} \int_{0}^{\infty} g_{1}\left(x \mid t_{1}\right) f\left(t_{1}, t\right) d t_{1} d t \\
& =\int_{0}^{\infty} g_{1}\left(x \mid t_{1}\right) \int_{0}^{\infty} e^{-s t} f\left(t_{1}, t\right) d t d t_{1} \\
& =\int_{0}^{\infty} g_{1}\left(x \mid t_{1}\right) \tilde{f}\left(t_{1}, s\right) d t_{1}
\end{aligned}
$$

where the function $f$ is zero for $t_{1}>t$, and $g_{1}(x \mid t)$ is given by (5). The Laplace transform of $f\left(t_{1}, t\right)$ with respect to $t$ is:

$$
\begin{aligned}
\tilde{f}\left(t_{1}, s\right)= & \int_{0}^{\infty} e^{-s t} f\left(t_{1}, t\right) d t \\
= & \frac{\mathrm{B}(\alpha-1, \beta)}{a \mathrm{~B}(\alpha, \beta)} \sum_{n=0}^{\infty} \frac{(2-\alpha-\beta)_{n}}{n !(2-\alpha)_{n}}\left(-\frac{s}{a} t_{1}\right)^{n} \\
& -\frac{\Gamma(2-\alpha) \Gamma(\alpha-1)}{a \Gamma(\alpha) \mathrm{B}(\alpha, \beta)} \sum_{n=0}^{\infty} \frac{(1-\beta)_{n}}{n !(\alpha)_{n}}\left(-\frac{s}{a} t_{1}\right)^{\alpha+n-1}
\end{aligned}
$$

where $(\alpha)_{n}=\alpha(\alpha+1) \cdots(\alpha+n-1)$ is the rising factorial. The moment generating func-

tion calculated from (C.1) is then:

$$
\begin{aligned}
\tilde{\psi}(x, s)= & \frac{1}{a}\left(1+\frac{\beta}{\alpha-1}\right) \sqrt{\frac{x}{\pi D_{1} u}} e^{\frac{u x}{\pi D_{1}}}\left[\sum_{n=0}^{\infty} \frac{(2-\alpha-\beta)_{n}}{n !(2-\alpha)_{n}} K_{\frac{1}{2}+n}\left(\frac{u x}{2 D_{1}}\right)\left(-\frac{s x}{a u}\right)^{n}\right. \\
& \left.-\frac{\Gamma(2-\alpha) \Gamma(\alpha-1)}{\Gamma(\alpha) \mathrm{B}(\alpha-1, \beta)}\left(\frac{s x}{a u}\right)^{\alpha-1} \sum_{n=0}^{\infty} \frac{(1-\beta)_{n}}{n !(\alpha)_{n}} K_{-\frac{1}{2}+\alpha+n}\left(\frac{u x}{2 D_{1}}\right)\left(-\frac{s x}{a u}\right)^{n}\right]
\end{aligned}
$$

where $K_{n}(\cdot)$ is the modified Bessel function of the second kind. The raw temporal moments of $\psi(x, t)$ can be obtained from the derivatives of (C.2):

$$
\mu_{T, n}(x)=\left.(-1)^{n} \frac{d^{n} \tilde{\psi}(x, s)}{d s^{n}}\right|_{s=0}
$$

Comparison between the particle tracking simulations and the approximating distribution shows that $\alpha>4$. Under this condition the second part of equation (C.2) does not contribute to the first three moments. Using (C.4) in combination with (62), (63) and (64) gives

$$
\begin{aligned}
\mu_{T, 0}= & \frac{1}{a u}\left(1+\frac{\beta}{\alpha-1}\right) \\
m_{T, 1}= & \frac{\alpha+\beta-2}{a(\alpha-2)}\left(\frac{2 D_{1}}{u^{2}}+\frac{x}{u}\right) \\
m_{T, 2}= & \frac{\alpha+\beta-2}{a^{2} u^{2}(\alpha-3)(\alpha-2)^{2}} \frac{D_{1}^{2}}{u^{2}}\left\{4\left[2 \alpha^{2}+2 \alpha(\beta-5)-3(\beta-4)\right]\right. \\
& \left.+2\left[6+\alpha(\beta-5)+\alpha^{2}\right] \frac{u x}{D_{1}}+\beta\left(\frac{u x}{D_{1}}\right)^{2}\right\}
\end{aligned}
$$


$\gamma_{T}=2 \sqrt{\frac{\alpha-3}{\alpha+\beta-2}}\left\{8 D_{1}^{3}\left[4 \alpha^{4}+\alpha^{3}(8 \beta-44)+2 \alpha^{2}\left(2 \beta^{2}-27 \beta+88\right)+\right.\right.$ $\left.\alpha\left(-10 \beta^{2}+115 \beta-304\right)+6\left(\beta^{2}-13 \beta+32\right)\right]+6 D_{1}^{2} u x\left[\alpha^{4}+\alpha^{3}(2 \beta-11)+\right.$ $\left.\alpha^{2}\left(\beta^{2}-9 \beta+44\right)+2 \alpha\left(\beta^{2}+4 \beta-38\right)-4 \beta^{2}+4 \beta+48\right]+$ $\left.6 \beta D_{1} u^{2} x^{2}\left[\alpha^{2}+\alpha(\beta-4)+4\right]+\beta u^{3} x^{3}(\alpha+2 \beta-2)\right\} /$ $\left\{(\alpha-4)\left[4 D_{1}^{2}\left(2 \alpha^{2}+2 \alpha(\beta-5)-3(\beta-4)\right)+2 D_{1} u x\left(\alpha^{2}+\alpha(\beta-5)+6\right)+\beta u^{2} x^{2}\right]^{3 / 2}\right\}$

At large distances from the injection point, $u x / D_{1} \gg 1$, equations (C.6), (C.7) and (C.8) converge to (67), (68) and (69), respectively.

\section{Acknowledgments}

The author would like to thank the School of Mechanical, Aerospace and Civil Engineering at The University of Manchester for financial support to carry out this research. Special thanks go also to Dr. Rui Ferreira for providing data for preliminary analyses at an early stage of the study. No data was used in producing this manuscript. All the numerical results presented in the figures are generated by solving the equations in the paper. The computer code used for the particle tracking simulations is available from the author upon request (andrea.bottacinbusolin@manchester.ac.uk).

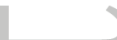

\section{References}

Aquino, T., A. Aubeneau, and D. Bolster (2015), Peak and tail scaling of breakthrough curves in hydrologic tracer tests, Adv. Water Resour., 78, 1-8, doi: 10.1016/j.advwatres.2015.01.016.

Aris, R. (1956), On the dispersion of a solute in a fluid flowing through a tube, Proc. $R$. Soc. Lond. A, 235(1200), 67-77, doi:10.1098/rspa.1956.0065.

Aubeneau, A. F., B. Hanrahan, D. Bolster, and J. L. Tank (2014), Substrate size and heterogeneity control anomalous transport in small streams, Geophys. Res. Lett., 41(23), 8335-8341, doi:10.1002/2014GL061838.

Battiato, I., and S. Rubol (2014), Single-parameter model of vegetated aquatic flows, Water Resour. Res., 50(8), 6358-6369, doi:10.1002/2013WR015065.

Bellin, A., and D. Tonina (2007), Probability density function of non-reactive solute concentration in heterogeneous porous formations, Journal of Contaminant Hydrology, 
94(1-2), 109-125, doi:10.1016/j.jconhyd.2007.05.005.

Bellin, A., D. Tonina, and A. Marzadri (2015), Breakthrough curve moments scaling in hyporheic exchange, Water Resour. Res., 51(2), 1353-1358, doi: 10.1002/2014WR016559.

Bencala, K. E., and R. A. Walters (1983), Simulation of solute transport in a mountain pool-and-riffle stream: A transient storage model, Water Resour. Res., 19(3), 718-724, doi:10.1029/WR019i003p00718.

Boano, F., A. I. Packman, A. Cortis, R. Revelli, and L. Ridolfi (2007), A continuous time random walk approach to the stream transport of solutes, Water Resour. Res., 43(10), W10,425, doi:10.1029/2007WR006062.

Boano, F., J. W. Harvey, A. Marion, A. I. Packman, R. Revelli, L. Ridolfi, and A. Wörman (2014), Hyporheic flow and transport processes: Mechanisms, models, and biogeochemical implications, Rev. Geophys., p. 2012RG000417, doi: 10.1002/2012RG000417.

Boso, F., F. P. J. de Barros, A. Fiori, and A. Bellin (2013), Performance analysis of statistical spatial measures for contaminant plume characterization toward risk-based decision making, Water Resour. Res., 49(6), 3119-3132, doi:10.1002/wrcr.20270.

Bottacin-Busolin, A., and A. Marion (2010), Combined role of advective pumping and mechanical dispersion on time scales of bed form-induced hyporheic exchange, Water Resour. Res., 46(8), W08,518, doi:10.1029/2009WR008892.

Bottacin-Busolin, A., A. Marion, T. Musner, M. Tregnaghi, and M. Zaramella (2011), Evidence of distinct contaminant transport patterns in rivers using tracer tests and a multiple domain retention model, Adv. Water Resour., 34(6), 737-746, doi: 10.1016/j.advwatres.2011.03.005.

Breugem, W. P., B. J. Boersma, and R. E. Uittenbogaard (2006), The influence of wall permeability on turbulent channel flow, Journal of Fluid Mechanics, 562, 35-72, doi: $10.1017 / \mathrm{S} 0022112006000887$.

Chandler, I. D., I. Guymer, J. M. Pearson, and R. van Egmond (2016), Vertical variation of mixing within porous sediment beds below turbulent flows, Water Resour. Res., 52(5), 3493-3509, doi:10.1002/2015WR018274.

Chanson, H. (2004), Hydraulics of Open Channel Flow, 2 edition ed., ButterworthHeinemann, Amsterdam. 
Chatwin, P. C. (1971), On the interpretation of some longitudinal dispersion experiments, J. Fluid Mech., 48(04), 689-702, doi:10.1017/S0022112071001800.

Chatwin, P. C. (1973), A calculation illustrating effects of the viscous sub-layer on longitudinal dispersion, Q. J. Mechanics Appl. Math, 26(4), 427-439, doi: 10.1093/qjmam/26.4.427.

Chatwin, P. C., D. M. Lewis, and P. J. Sullivan (1995), Turbulent dispersion and the beta distribution, Environmetrics, 6(4), 395-402, doi:10.1002/env.3170060408.

Chen, C.-1. (1991), Unified Theory on Power Laws for Flow Resistance, Journal of Hydraulic Engineering, 117(3), 371-389, doi:10.1061/(ASCE)0733-9429(1991)117:3(371).

Day, T. J. (1975), Longitudinal dispersion in natural channels, Water Resour. Res., 11(6), 909-918, doi:10.1029/WR011i006p00909.

Drummond, J. D., T. P. Covino, A. F. Aubeneau, D. Leong, S. Patil, R. Schumer, and A. I. Packman (2012), Effects of solute breakthrough curve tail truncation on residence time estimates: A synthesis of solute tracer injection studies, J. Geophys. Res., 117(G3), G00N08, doi:10.1029/2012JG002019.

Elder, J. W. (1959), The dispersion of marked fluid in turbulent shear flow, J. Fluid Mech., 5(04), 544-560, doi:10.1017/S0022112059000374.

Ferreira, R. M., L. M. Ferreira, A. M. Ricardo, and M. J. Franca (2010), Impacts of sand transport on flow variables and dissolved oxygen in gravel-bed streams suitable for salmonid spawning, River Res. Applic., 26(4), 414-438, doi:10.1002/rra.1307.

Fischer, H., E. J. List, and H. B. Fischer (1979), Mixing in inland and coastal waters, Academic Press, New York.

Fischer, H. B. (1966), Longitudinal dispersion in laboratory and natural streams, Tech. Rep. KH-R-12, California Institute of Technology, Pasadena, California.

Fischer, H. B. (1967), The mechanics of dispersion in natural streams, J. Hydr. Eng. Div.ASCE, 93(6), 187-216.

Fischer, H. B. (1968), Dispersion predictions in natural streams, J. Sanitary Eng. Div., 94(5), 927-944.

Gardiner, C. (2009), Stochastic Methods: A Handbook for the Natural and Social Sciences, 4th ed. 2009 edition ed., Springer, Berlin.

Godfrey, R. G., and B. J. Frederick (1963), Dispersion in natural streams, USGS Unnumbered Series, U.S. Geological Survey, Washington, D.C. 
González-Pinzón, R., R. Haggerty, and M. Dentz (2013), Scaling and predicting solute transport processes in streams, Water Resour. Res., 49(7), 4071-4088, doi: 10.1002/wrcr.20280.

Gooseff, M. N., M. A. Briggs, K. E. Bencala, B. L. McGlynn, and D. T. Scott (2013), Do transient storage parameters directly scale in longer, combined stream reaches? Reach length dependence of transient storage interpretations, J. Hydrol., 483, 16-25, doi:10.1016/j.jhydrol.2012.12.046.

Haggerty, R., S. A. McKenna, and L. C. Meigs (2000), On the late-time behavior of tracer test breakthrough curves, Water Resour. Res., 36(12), 3467-3479, doi: 10.1029/2000WR900214.

Hays, J. R. (1966), Mass transport mechanisms in open-channel flow, Tech. rep., Dept. of Civil Engineering, Vanderbilt University, Nashville, Tenn.

Hester, E. T., M. B. Cardenas, R. Haggerty, and S. V. Apte (2017), The importance and challenge of hyporheic mixing, Water Resour. Res., pp. n/a-n/a, doi: 10.1002/2016WR020005.

Jackson, T. R., R. Haggerty, and S. V. Apte (2013), A fluid-mechanics based classification scheme for surface transient storage in riverine environments: quantitatively separating surface from hyporheic transient storage, Hydrol. Earth Syst. Sci., 17(7), 2747-2779, doi:10.5194/hess-17-2747-2013.

Jackson, T. R., S. V. Apte, and R. Haggerty (2014), Effect of multiple lateral cavities on stream solute transport under non-Fickian conditions and at the Fickian asymptote, $J$. Hydrol., 519, Part B, 1707-1722, doi:10.1016/j.jhydrol.2014.09.036.

Kenney, J. F., and E. S. Keeping (1947), Mathematics of statistics, Van Nostrand, googleBooks-ID: UdlLAAAAMAAJ.

Kitanidis, P. K. (1994), Particle-tracking equations for the solution of the advectiondispersion equation with variable coefficients, Water Resour. Res., 30(11), 3225-3227, doi:10.1029/94WR01880.

Kokoska, S., and D. Zwillinger (2000), Standard probability and statistics tables and formulae, CRC Press.

Leu, J. M., H. C. Chan, L.-F. Tu, Y. Jia, and S. Y. Wang (2009), Velocity distribution of non-Darcy flow in a porous medium, J. Mech., 25(1), 49-58, doi: $10.1017 / \mathrm{S} 1727719100003592$. 
Marion, A., M. Zaramella, and A. Bottacin-Busolin (2008a), Solute transport in rivers with multiple storage zones: The STIR model, Water Resour. Res., 44(10), W10,406, doi:10.1029/2008WR007037.

Marion, A., A. I. Packman, M. Zaramella, and A. Bottacin-Busolin (2008b), Hyporheic flows in stratified beds, Water Resour. Res., 44(9), W09,433, doi: 10.1029/2007WR006079.

Musner, T., A. Bottacin-Busolin, M. Zaramella, and A. Marion (2014), A contaminant transport model for wetlands accounting for distinct residence time bimodality, Journal of Hydrology, 515, 237-246, doi:10.1016/j.jhydrol.2014.04.043.

Nepf, H. M. (2012), Flow and Transport in Regions with Aquatic Vegetation, Annual Review of Fluid Mechanics, 44(1), 123-142, doi:10.1146/annurev-fluid-120710-101048.

Nordin, C. F., and G. V. Sabol (1974), Empirical data on longitudinal dispersion in rivers, USGS Numbered Series 74-20, U.S. Geological Survey.

Nordin, C. F., and B. M. Troutman (1980), Longitudinal dispersion in rivers: The persistence of skewness in observed data, Water Resour. Res., 16(1), 123-128, doi: 10.1029/WR016i001p00123.

Pannone, M. (2012), On the exact analytical solution for the spatial moments of the crosssectional average concentration in open channel flows, Water Resources Research, 48(8), n/a-n/a, doi:10.1029/2011WR011665.

Pedersen, F. B. (1977), Prediction of longitudinal dispersion in natural streams, Tech. Rep. Ser. Pap. 14, Inst. of Hydrodyn. and Hydraul. Eng., Tech. Univ. of Denmark, Lyngby, Denmark.

Platen, E. (1995), On weak implicit and predictor-corrector methods, Mathematics and Computers in Simulation, 38(1), 69-76, doi:10.1016/0378-4754(93)E0068-G.

Platen, E., and N. Bruti-Liberati (2010), Numerical solution of stochastic differential equations with jumps in finance, Stochastic Modelling and Applied Probability, vol. 64, Springer Berlin Heidelberg, Berlin, Heidelberg.

Rodi, W. (1993), Turbulence Models and Their Application in Hydraulics: A State-of-theArt Review, 1 edition ed., Routledge, Rotterdam.

Rubol, S., I. Battiato, and F. P. J. de Barros (2016), Vertical dispersion in vegetated shear flows, Water Resour. Res., 52(10), 8066-8080, doi:10.1002/2016WR018907.

Sayre, W., and F. Chang (1968), A laboratory investigation of open-channel dispersion processes for dissolved, suspended, and floating dispersants, USGS Numbered Series 
433-E, U.S. Geological Survey.

Schmid, B. (2002), Persistence of skewness in longitudinal dispersion data: Can the dead zone model explain it after all?, J. Hydraul. Eng.-ASCE, 128(9), 848-854, doi: 10.1061/(ASCE)0733-9429(2002)128:9(848).

Seo, I. W., and K. O. Baek (2004), Estimation of the Longitudinal Dispersion Coefficient Using the Velocity Profile in Natural Streams, Journal of Hydraulic Engineering, 130(3), 227-236, doi:10.1061/(ASCE)0733-9429(2004)130:3(227).

Shucksmith, J. D., J. B. Boxall, and I. Guymer (2010), Effects of emergent and submerged natural vegetation on longitudinal mixing in open channel flow, Water Resources Research, 46(4), n/a-n/a, doi:10.1029/2008WR007657.

Taylor, G. (1953), Dispersion of soluble matter in solvent flowing slowly through a tube, P. Roy. Soc. Lond. A Mat., 219(1137), 186-203, doi:10.1098/rspa.1953.0139.

Taylor, G. (1954), The dispersion of matter in turbulent flow through a pipe, Proc. R. Soc. Lond. A Mat., 223(1155), 446-468, doi:10.1098/rspa.1954.0130.

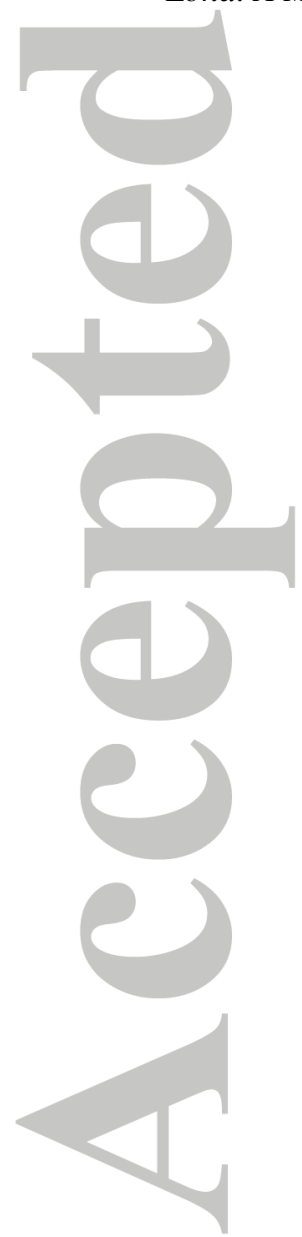


Figure 1.

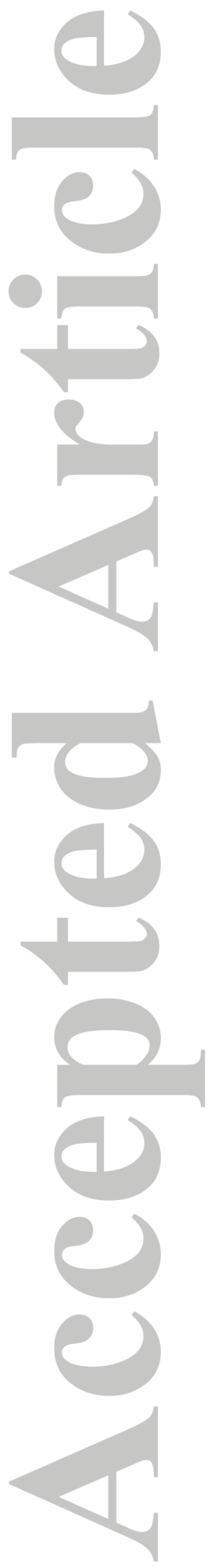

This article is protected by copyright. All rights reserved. 
(a)

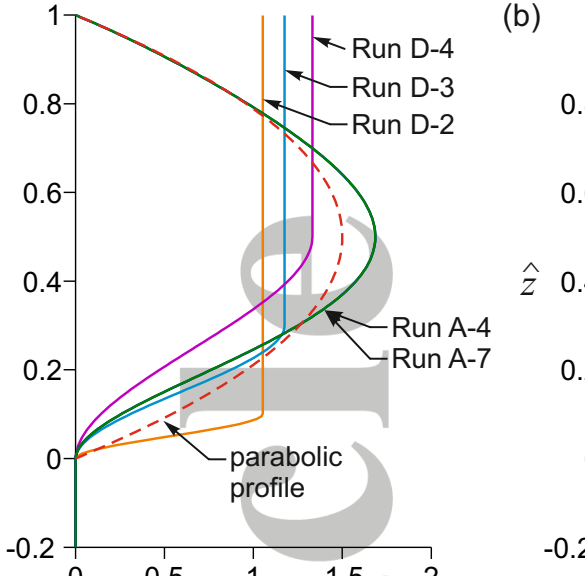

(b)

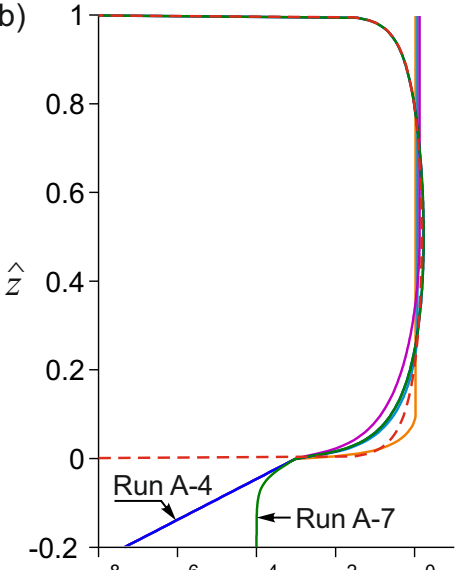


Figure 2.

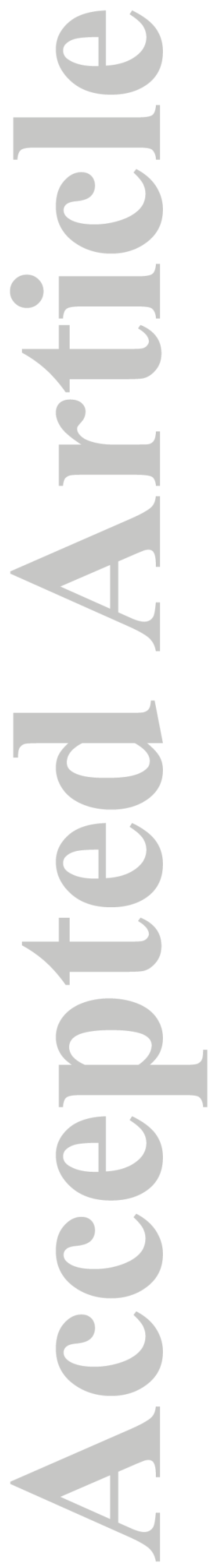

This article is protected by copyright. All rights reserved. 
Figure 3.

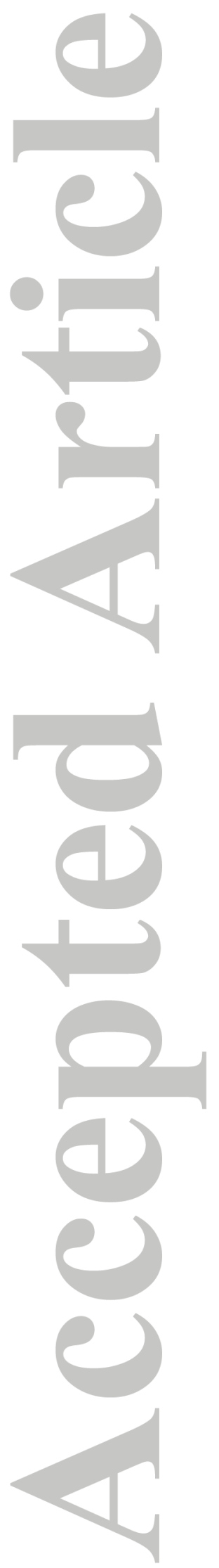

This article is protected by copyright. All rights reserved. 


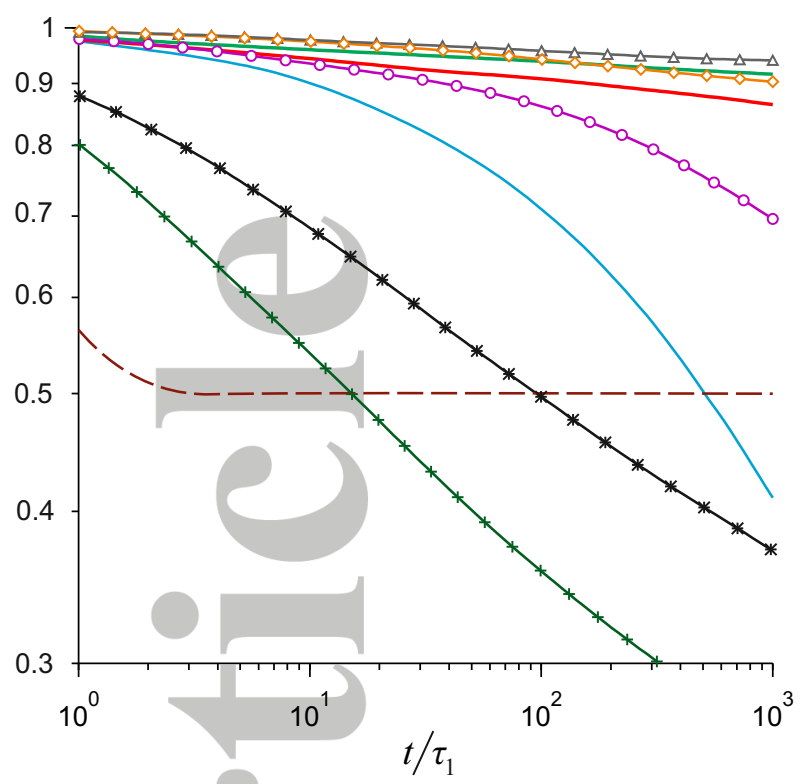

(b)
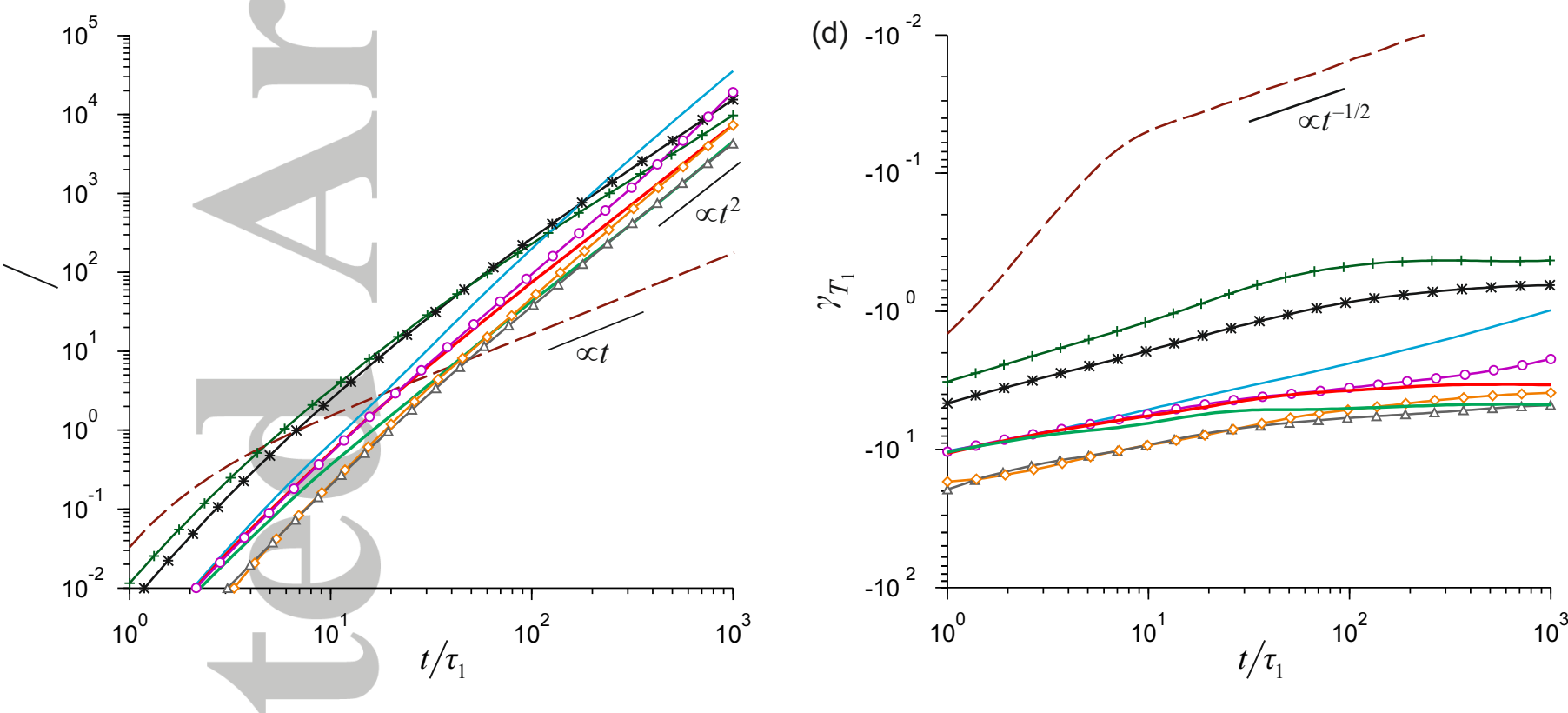

Run A-4 $\quad \longrightarrow$ Run A-7 
Figure 4.

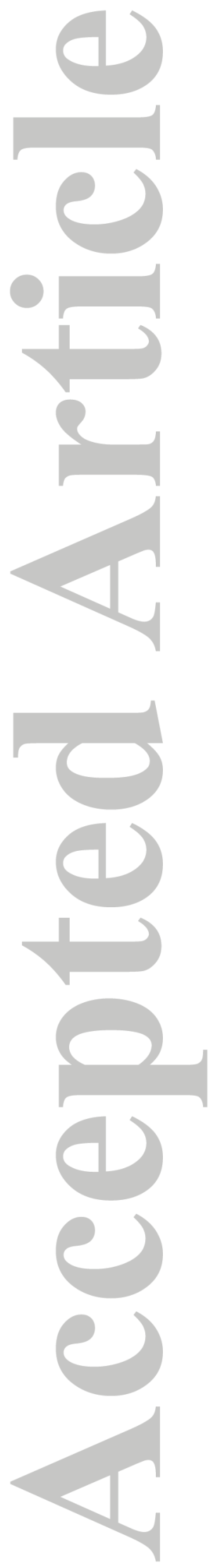

This article is protected by copyright. All rights reserved. 
Figure 5.

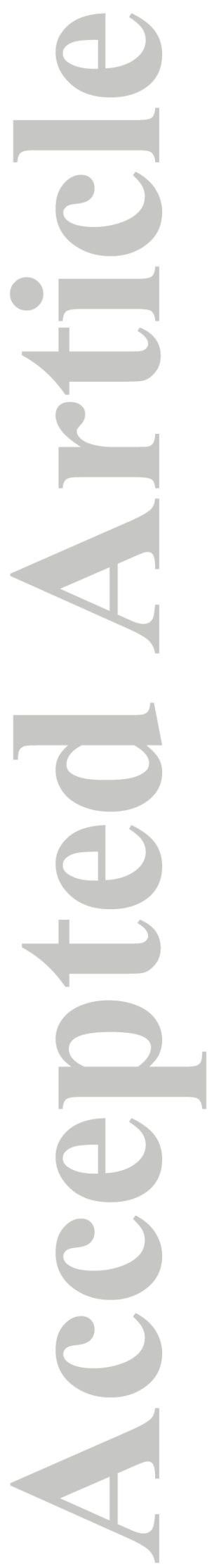

This article is protected by copyright. All rights reserved. 
Figure 6.

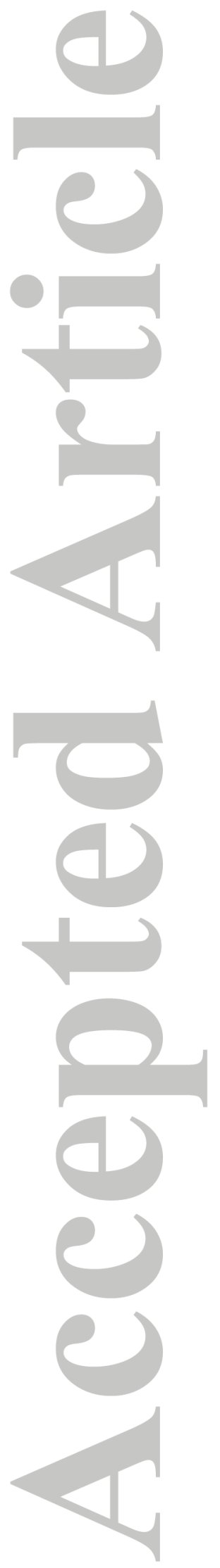

This article is protected by copyright. All rights reserved. 


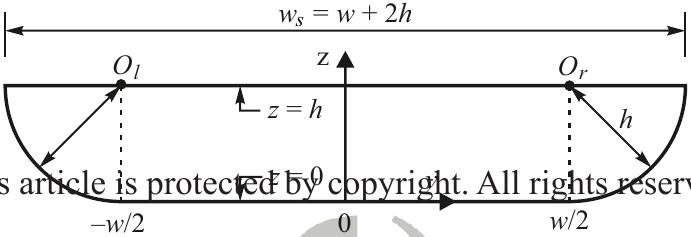


Figure 7.
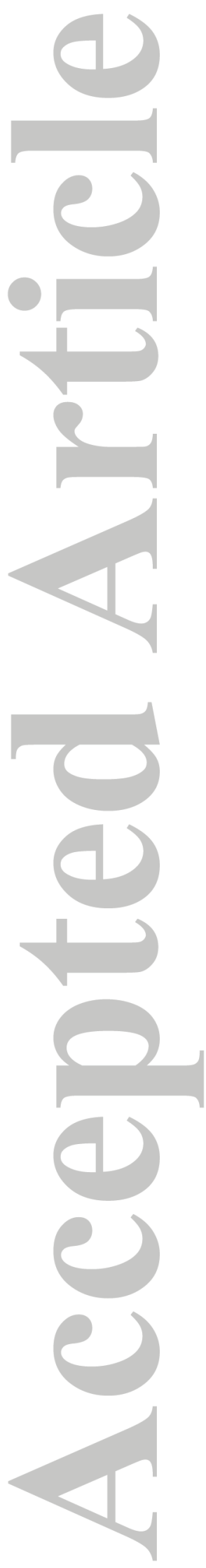

This article is protected by copyright. All rights reserved. 
Figure 8.

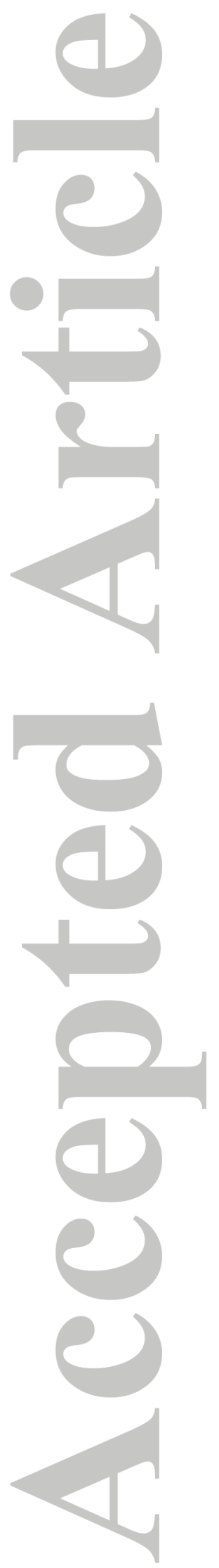

This article is protected by copyright. All rights reserved. 

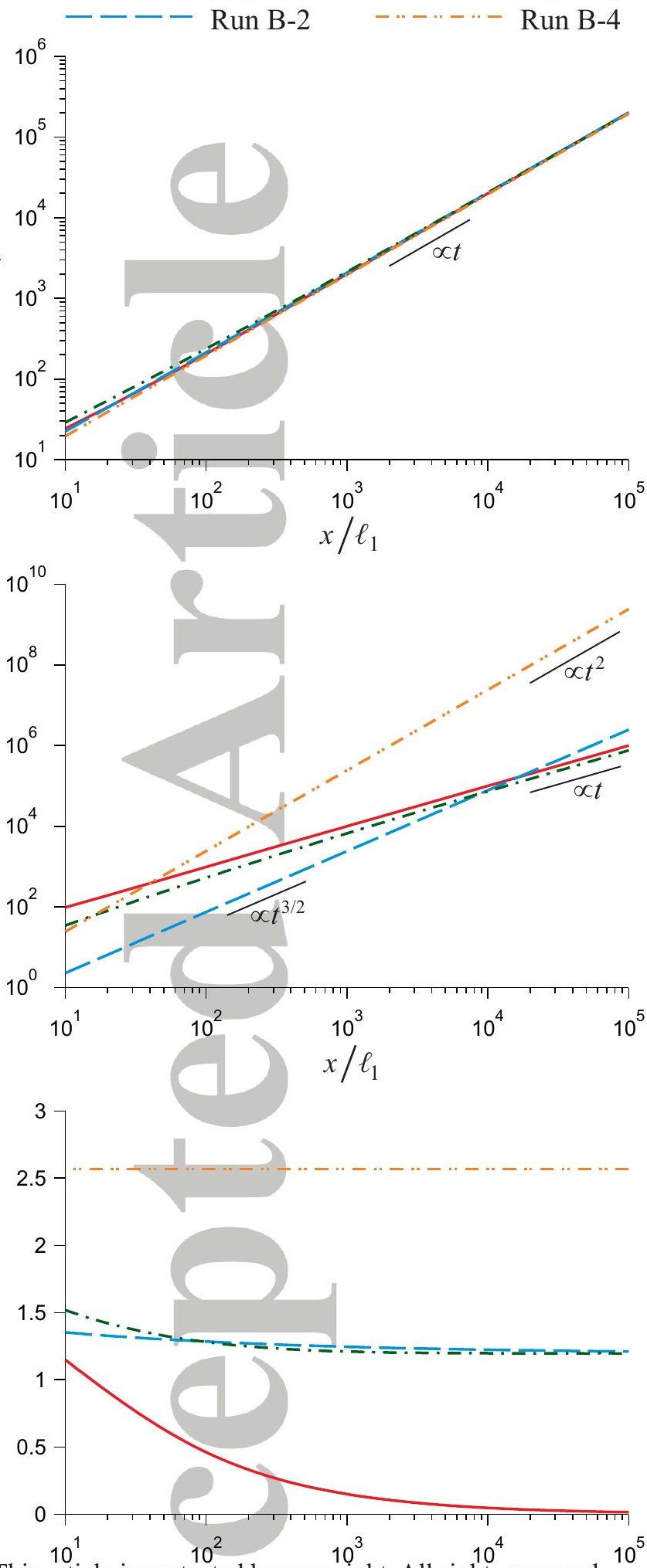

This article is protected by copyright. All rights reserved. 
Figure 9.

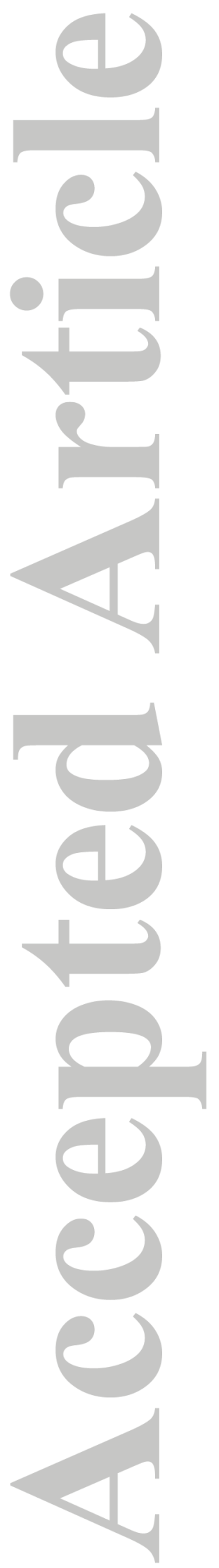

This article is protected by copyright. All rights reserved. 

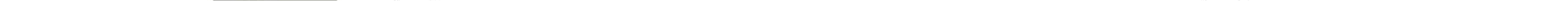